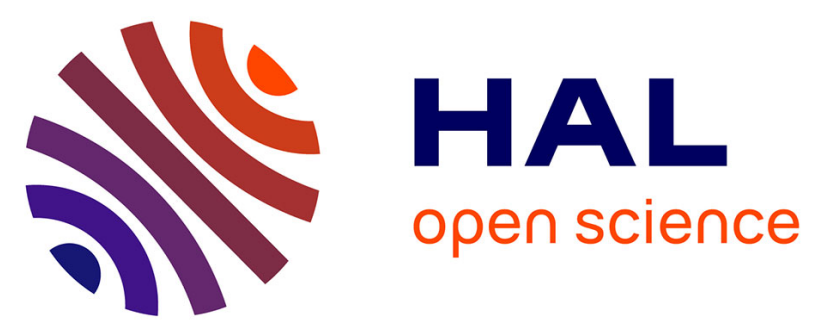

\title{
Radiative, collisional atomic and Stark broadening data for Ar XII and Ar XIV ions: quantum mechanical calculations
}

Rihab Aloui, Haykel Elabidi, Sylvie Sahal-Bréchot

\section{To cite this version:}

Rihab Aloui, Haykel Elabidi, Sylvie Sahal-Bréchot. Radiative, collisional atomic and Stark broadening data for Ar XII and Ar XIV ions: quantum mechanical calculations. Journal of Quantitative Spectroscopy and Radiative Transfer, 2019, 239, pp.106675. 10.1016/j.jqsrt.2019.106675 . hal-02340062

\section{HAL Id: hal-02340062 \\ https://hal.sorbonne-universite.fr/hal-02340062}

Submitted on 30 Oct 2019

HAL is a multi-disciplinary open access archive for the deposit and dissemination of scientific research documents, whether they are published or not. The documents may come from teaching and research institutions in France or abroad, or from public or private research centers.
L'archive ouverte pluridisciplinaire HAL, est destinée au dépôt et à la diffusion de documents scientifiques de niveau recherche, publiés ou non, émanant des établissements d'enseignement et de recherche français ou étrangers, des laboratoires publics ou privés. 


\title{
Radiative, collisional atomic and Stark broadening data for Ar XII and Ar XIV ions: quantum mechanical calculations
}

\author{
Rihab Aloui ${ }^{\mathrm{a}}$, Haykel Elabidi ${ }^{\mathrm{b}, \mathrm{c}, *}$, Sylvie Sahal-Bréchot ${ }^{\mathrm{d}}$ \\ ${ }^{a}$ Laboratoire de Spectroscopie et Dynamique Moléculaire, École Nationale Supérieure d'Ingénieurs de Tunis, University of Tunis, Tunisia \\ ${ }^{b}$ Department of Physics, Deanship of the Common First Year, Umm Al-Qura University, Makkah Almukarramah, Saudi Arabia \\ ${ }^{c}$ Laboratoire de Spectroscopie et Dynamique Moléculaire, GRePAA, Faculté des Sciences de Bizerte, University of Carthage, Tunisia \\ ${ }^{d}$ Sorbonne Université, Observatoire de Paris, Université PSL, CNRS, LERMA, F-92190 Meudon, France
}

\begin{abstract}
We present in this work Stark broadening calculations for $12 \mathrm{Ar}$ XII and $12 \mathrm{Ar}$ XIV lines. We present also their radiative atomic and collision data (energy levels, radiative decay rates, line strengths, oscillator strengths and collision strengths). Our Stark broadening data have been obtained using our quantum method in the impact approximation frame and including fine structure effects in the structure study. Our results are provided for the range of temperatures from $5 \times 10^{5}$ to $5 \times 10^{6} \mathrm{~K}$ of interest for plasmas investigations, and at electron density $N_{e}=10^{18} \mathrm{~cm}^{-3}$. The radiative atomic data have been calculated using the UCL code SUPERSTRUCTURE. The collision strengths have been calculated using the two UCL codes DISTORTED WAVE and JAJOM. Good agreement have been found between our atomic results and other theoretical and experimental ones. Our Stark broadening data are the first to be published, so no comparisons have been performed for them.
\end{abstract}

Keywords: line profiles- Stark line broadening- white dwarfs- atmospheres- atomic data.

\section{Introduction}

In the past, trace elements had no significance for the investigation of stellar spectra. Nowadays, with the development of satellite-born spectrographs and space astronomy in general, analysis of the spectrum of some astrophysical objects show spectral lines of different highly charged ions of trace elements. For example, new Ar VI [1] and Ar VII [2] lines have been discovered in hot stars and white dwarfs. Werner et al. [3] detected Br VI, Sb V and Sb VI ions for the first time in heliumrich white dwarfs. The analysis of the spectra needs atomic and line broadening data: for the evaluation of the abundance of $\mathrm{Ba} \mathrm{V}, \mathrm{Ba}$ VI, and Ba VII [4] and of the Se V, Sr IV-VII, Te VI, and I VI ions [5] in the white dwarfs' G191-B2B and RE 0503-289, the authors calculated the oscillator strengths of the emitted lines and included them in their atmosphere-model calculations. Nikiforov et al. [6] have used Stark broadening data of hydrogenic and non-hydrogenic lines to determine the electron density in atmospheric pressure plasma jets. Rauch et al. [1] showed that the lack of atomic and line broadening parameters of many elements presents an obstacle for plasma research, and concluded that the difference between their resonance doublets line profiles of N V, O VI, and S VI and the observation can be explained by the missing of line broadening tables. Better atomic and line broadening data will improve spectral analyses in the future. Barstow et al. [7] have shown that models which neglect the opacity of heavy elements are not

\footnotetext{
*Corresponding author. Tel.: +966564518933; fax: +966564518933

Email address: haelabidi@uqu.edu.sa (Haykel Elabidi)
}

well suited for the analysis of $\mathrm{DA}(\mathrm{O})$ white dwarfs. Werner [8] showed that when introducing $\mathrm{C}, \mathrm{N}$, and $\mathrm{O}$ (considered before without significance) and their detailed Stark broadening in the model, the precedent problem vanishes. Consequently, atomic, collision and line broadening data of these ions are prerequisite for the atmosphere-model calculations.

Spectral lines of highly charged argon ions (Ar IX-XVIII) were observed by Zhang et al. [9] when analysing highresolution spectra in the UV, EUV and X-ray regions obtained for stellar and other astrophysical sources by several space missions such as Chandra, and XMM-Newton. Wang et al. [10] identified and analysed some lines of argon ions from Ar XII to Ar XVI obtained with Texas Experimental Tokamak (TEXT) for temperature about $2.4 \times 10^{6}-4 \times 10^{6} \mathrm{~K}$. For Ar XII, the line is $2 \mathrm{~s}^{2} 2 \mathrm{p}^{3}\left({ }^{2} \mathrm{D}_{5 / 2}\right)-2 \mathrm{~s} 2 \mathrm{p}^{4}\left({ }^{2} \mathrm{D}_{5 / 2}\right)$ at $193.7 \AA$ and for Ar XIV, the line is $2 \mathrm{~s}^{2} 2 \mathrm{p}\left({ }^{2} \mathrm{P}_{3 / 2}\right)-2 \mathrm{~s} 2 \mathrm{p}^{2}\left({ }^{2} \mathrm{D}_{3 / 2,5 / 2}\right)$ at $257.5 \AA$ A. Lepson et al. [11] performed experiments on the Ar IX-Ar XVI ions with the Lawrence Livermore electron beam ion traps EBIT-I and EBITII, and identified 79 transitions with their wavelengths. Other observations of the spectra of $\mathrm{Ar} \mathrm{X}$ to $\mathrm{Ar} \mathrm{XV}$ in the UV range (140-300 Å) were performed in Fawcett et al. [12]. Connerade et al. [13] observed about 60 lines of argon ions from Ar IX to Ar XVIII in the X-ray range (20-40 $⿱$ ) .

The atomic and collision data of Ar XII and Ar XIV ions were studied a long time ago. Since the 1960s, Ar XII lines were studied $[14,15,16]$ in $\vartheta$-pinch UV light sources, for instance, Fawcett and Gabriel [15] identified the resonance lines of argon XI and XII in the wavelength range 150 to $200 \AA$. A beam-foil spectroscopy of highly ionized Argon was performed in Buchet et al. [17], where more than thirty observed lines 
were identified as hydrogenic transitions in Ar X-XV. Oscillator strengths, radiative decay rates, line intensities and electron collision strengths were calculated for the nitrogen isoelectronic sequence (including Ar XII) in Bhatia et al. [18] and for boron isoelectronic sequence (including Ar XIV) in Bhatia et al. [19]. Collision strengths in Bhatia et al. $[18,19]$ were calculated at one electron energy. Zhang and Sampson [20] used the relativistic distorted-wave method to provide oscillator strengths and collision strengths for the $\Delta n=0$ transitions with $n=2$ in $\mathrm{Ar}$ XII and N-like other ions with $12 \leq Z \leq 92$. Eissner et al. [21] performed the same calculations as in [18, 19] but collision strengths were provided for five incident electron energies. Zhang et al. [9] calculated the energies of the 204 levels of the Ar XIV ion in the relativistic configuration interaction (RCI) formalism using multi-configuration Dirac-Fock (MCDF) wavefunctions. Rynkun et al. [22] calculated the energies and E1, M1, E2, M2 transition rates for states of the configurations $2 \mathrm{~s}^{2} 2 \mathrm{p}, 2 \mathrm{~s} 2 \mathrm{p}^{2}$ and $2 \mathrm{p}^{3}$ in boron-like ions between N III and Zn XXVI including Ar XIV. Wang et al. [23] improved the work of Bhatia et al. [18] and used combined relativistic configuration interaction and many-body perturbation calculations to provide energies and transitions rates of Ar XII and (other N-like ions up to Zn XXIV) including configurations with $n=2,3$ yielding 359 fine structure levels. The authors in Wang et al. [23] expected that their data sets would be useful for the identification and interpretation of observed spectra, and for modelling and diagnosing of astrophysical and fusion plasmas. Transitions of type $2 \mathrm{~s}^{2} 2 \mathrm{p}^{3}-2 \mathrm{~s} 2 \mathrm{p}^{4}$ in Ar XII were prominent in tokamak plasmas and $2 \mathrm{~s}^{2} 2 \mathrm{p}^{3}-2 \mathrm{~s}^{2} 2 \mathrm{p}^{2} 3 \mathrm{~d}$ transitions were typically intense in high density laser-produced plasmas [18]. Transitions in B-like Ar XIV were observed in the spectra from tokamak plasmas [19]. Mattioli et al. [24] analysed the experimental spectra of argon in the wavelength range 23-34 $\AA$ from jet tokamak plasmas and simulated them using collisional-radiative models constructed for Ar XII-Ar XVI ions. The argon ions were injected as impurities into tokamak plasmas [9].

No line broadening results have been found in the literature for the ions Ar XII and Ar XIV. The present work is an effort to provide Stark broadening data for these two ions using our quantum method. The expression giving the electron impact broadening of spectral lines was established in Elabidi et al. $[25,26]$, where configuration interactions and relativistic effects for the atomic structure were taken into account in intermediate coupling. This method was applied many times in the last ten years: the first applications of our method were performed for the Be-like [26] and Li-like [27] ions, where extensive comparisons were done with many theoretical and experimental results. Satisfactory agreement was found. The Stark broadening of Si IV was calculated using our method in Elabidi et al. [28] and comparisons with the experimental results of Bukvić et al. [29] showed acceptable agreements. Extensive study of the influence of strong collisions on Stark broadening was performed in [30] for the calculations of Ar VII line widths. Recently, this method was used by Elabidi and Sahal-Bréchot [31] to provide scaling of Stark broadening with electron temperature and by Elabidi and Sahal-Bréchot [32] to provide scaling with electron density. Calculations of Stark widths of some Ar VIII and Ar
IX lines of astrophysical interest were performed in Aloui et al. [33], where a comparison between the importance of Stark and Doppler broadening in the atmospheric conditions of DO white dwarfs was performed.

In the present paper, we use our quantum mechanical method for calculating electron impact widths of Ar XII and Ar XIV lines for electron temperature ranging from $5 \times 10^{5}$ to $5 \times 10^{6} \mathrm{~K}$ and electron density $N_{e}=10^{18} \mathrm{~cm}^{-3}$. We provide also energy levels, radiative decay rates, oscillator strengths, line strengths and collision strengths of the considered ions. These parameters are used in our line broadening calculation. We have used the University College London (UCL) packages: the SUPERSTRUCTURE code [34] for the structure study, and the codes DISTORTED WAVE [35] and JAJOM [36] for the collision study. We find that our atomic and collision data are close to other theoretical and experimental results. The acceptable agreement of atomic and collision data indicates that our line broadening results have a sufficient accuracy. We expect that our calculations could fill the lack of Ar XII and Ar XIV line broadening data.

\section{Outline of our line broadening method}

We have used our quantum mechanical method to calculate Ar XII and Ar XIV electron Stark broadening, where the impact approximation has been assumed. More details can be found in Elabidi et al. [25, 26]. The expression of the full width at halfmaximum $W$ is valid at the temperatures and densities studied and it is given by:

$$
\begin{aligned}
W= & 2 N\left(\frac{\hbar}{m}\right)^{2}\left(\frac{2 m \pi}{k_{B} T}\right)^{\frac{1}{2}} \\
& \times \int_{0}^{\infty} \Gamma_{w}(\varepsilon) \exp \left(-\frac{\varepsilon}{k_{B} T}\right) d\left(\frac{\varepsilon}{k_{B} T}\right),
\end{aligned}
$$

where the integration is over the Maxwellian velocity distribution. $k_{B}$ is the Boltzmann constant, $m$ the electron mass, $T$ and $N$ are the electron temperature and density, respectively. $\varepsilon$ is the incident electron energy ans $\Gamma_{w}(\varepsilon)$ is given by:

$$
\begin{aligned}
\Gamma_{w}(\varepsilon)= & \sum_{J_{i}^{T} J_{f}^{T} l K_{i} K_{f}} \frac{\left[K_{i}, K_{f}, J_{i}^{T}, J_{f}^{T}\right]}{2} \\
& \times\left\{\begin{array}{ccc}
J_{i} & K_{i} & l \\
K_{f} & J_{f} & 1
\end{array}\right\}^{2}\left\{\begin{array}{lll}
K_{i} & J_{i}^{T} & s \\
J_{f}^{T} & K_{f} & 1
\end{array}\right\}^{2} \\
& \times\left[1-\left(\operatorname{Re}\left(\mathbb{S}_{i}\right) \operatorname{Re}\left(\mathbb{S}_{f}\right)+\operatorname{Im}\left(\mathbb{S}_{i}\right) \operatorname{Im}\left(\mathbb{S}_{f}\right)\right)\right]
\end{aligned}
$$

where $L_{j}+S_{j}=J_{j}, J_{j}+l=K_{j}$ and $K_{j}+s=J . S$ and $L$ designate the spin and orbital angular momenta of the target and $l$ is the electron orbital momentum. $\mathbb{S}_{i}\left(\mathbb{S}_{f}\right)$ represent the scattering matrix elements for the initial (final) levels in intermediate coupling, $\operatorname{Re}(\mathbb{S})$ and $\operatorname{Im}(\mathbb{S})$ are the real and the imaginary parts of the $\mathbb{S}$-matrix. The terms in braces represent the $6-\mathrm{j}$ symbols and we make use of the notation $[x, y, \ldots]=(2 x+1)(2 y+1) \ldots$ Both $\mathbb{S}_{i}$ and $\mathbb{S}_{f}$ computed using the same incident electron 
energy $\varepsilon=m v^{2} / 2$. The expression (1) of electron impact width $W$ includes relativistic effects of the target in intermediate coupling. The atomic structure calculations are performed using the SUPERSTRUCTURE (SST) code [34]. The radial wave functions are determined by diagonalization of the non relativistic Hamiltonian using orbitals calculated in a scaled Thomas-Fermi-Dirac Amaldi (TFDA) potential. The potential depends on scaling parameters $\lambda_{l}$ that have been obtained by a self-consistent energy minimization on the term energies included in our calculations. This code takes into account configuration interactions and relativistic (one-body, spin-orbit, mass and Darwin) corrections. These effects are introduced according to the Breit-Pauli approach [37]. The SST code provides energy levels, radiative data (decay rates, oscillator strengths, line strengths, ...) and the Term Coupling Coefficients (TCC) which will be used after by the JAJOM code. The electron-ion scattering calculations in $L S$ coupling are performed using the DISTORTED WAVE (DW) code [35] to produce the reactance matrices $\Re$ between terms ${ }^{2 S+1} L$. The JAJOM code [36] is used to treat the scattering calculations in intermediate coupling: it makes use of the TCC -supplied by the code SST- to transform the $\Re$ matrices (provided by DW) from $L S$ coupling to the intermediate one and calculates fine structure collision strengths.

Many years ago, the three codes SST/DW/JAJOM were used until the step of providing collision strengths. We have adapted them to our line broadening expression (1): The code JAJOM was transformed into the code JAJPOLARI (Dubau, unpublished results) to produce the reactance matrices $\mathfrak{R}$. Our code RtoS (Elabidi \& Dubau, unpublished results) calculate the scattering matrices $\mathbb{S}$ from the $\mathfrak{R}$ matrices and their real and imaginary parts in an adequate order to be used as input data suitable for the formula $(2) \operatorname{Re}(\mathbb{S})$ and $\operatorname{Im}(\mathbb{S})$ are given by:

$$
\operatorname{Re}(\mathbb{S})=\left(1-\mathfrak{R}^{2}\right)\left(1+\mathfrak{R}^{2}\right)^{-1}, \operatorname{Im}(\mathbb{S})=2 \mathfrak{R}\left(1+\mathfrak{R}^{2}\right)^{-1}
$$

\section{Results and discussions}

\subsection{Structure and radiative data}

For Ar XII, 6 configurations have been used in our study: $1 \mathrm{~s}^{2}$ $\left(2 s^{2} 2 p^{3}, 2 s 2 p^{4}, 2 p^{5}, 2 s^{2} 2 p^{2} 3 s, 2 s^{2} 2 p^{2} 3 p\right.$ and $\left.2 s^{2} 2 p^{2} 3 d\right)$. This set of configurations gives rise to 34 terms and 72 fine structure levels. For Ar XIV, we have used 8 configurations: $1 \mathrm{~s}^{2}$ $\left(2 s^{2} 2 p, 2 s p^{2}, 2 p^{3}, 2 s^{2} 3 s, 2 s^{2} 3 p, 2 s^{2} 3 d, 2 s 2 p 3 s\right.$, and $\left.2 s 2 p 3 p\right)$. These configurations give rise to 23 terms and 45 fine structure levels. The code SST provides also the scaling parameters: $\lambda_{s}=1.3425, \lambda_{p}=1.2481$ and $\lambda_{d}=1.3268$ for Ar XII, and $\lambda_{s}=1.4216, \lambda_{p}=1.2885$ and $\lambda_{d}=1.5300$ for Ar XIV. In Table 1, we present our energy levels $E\left(\right.$ in $\mathrm{cm}^{-1}$ ) for the $\mathrm{Ar}$ XII and compare them with NIST [38], with the experimental results (Edl) of Edlén [39] and with those calculated by Bhatia et al. [18] using the code SST but with low angular momenta $l<8\left(\mathrm{SST}_{7}\right)$. The difference between our energies and those of the references $[18,38,39]$ varies from nearly $0 \%$ to $4 \%$ with an average value less than $2 \%$. In Table 2 , we present the energy levels for Ar XIV expressed in $\mathrm{cm}^{-1}$ and compared with NIST [38] values, with the results calculated by Liang et al. [40] using the AUTOSTRUCTURE (AS) code [41] and with those of Aggarwal et al. [42] using the General Purpose Relativistic Atomic Structure Package (GRASP) of Dyall et al. [43]. As it is shown in Table 2, the percentage errors varies from $0.28 \%$ to nearly $5 \%$ (the value $5 \%$ is only with NIST values and for two levels $2 \mathrm{~s} 2 \mathrm{p} 3 \mathrm{~s}{ }^{2} \mathrm{P}_{1 / 2,3 / 2}^{\mathrm{o}}$ ). The averaged value is about $2 \%$. In the cases where we found an error of about $5 \%$ with NIST, there is a good agreement with the AS and the GRASP results (less than $0.5 \%$ ).

We present in Table 3 the radiative data for some Ar XII lines up to the level 15. Our radiative decay rates $A_{i j}$ and weighted oscillator strengths $g f$ are compared to the relativistic distortedwave calculations (ZS99) of Zhang and Sampson [20], to the many-body perturbation theory (MBPT) calculations (M97) obtained by Merkelis et al. [44] and reported in Eissner et al. [21], and to the calculations of Bhatia et al. [18] who used the code SUPERSTRUCTURE $\left(\mathrm{SST}_{7}\right)$ with $l \leq 7$. Our $g f$ and $A_{i j}$ values are very close to those of Bhatia et al. [18] (about 2\%), but the comparison with (ZS99) and (M97) results gives a higher error but still acceptable: about $14 \%$. The error for $A_{Z S 99}$ of the transition $(10-1)$ can reach $84 \%$. If we remove this value, the average error becomes about $9 \%$. We provide also some line strengths $(S)$, but no comparisons have been performed, because we did not find other results to compare them. The radiative data for some Ar XIV lines (up to level 14) have been reported in Table 4. We compare our results to the Multiconfiguration Dirac-Hartree-Fock (MCDHF) method of Grant [45] performed by Rynkun et al. [22] using the code GRASP2K [46]. The differences between the two results are about $16 \%$ for $A_{i j}$, approximately equal to $14 \%$ for $g f$ and $S$ values. The error values can reach $50 \%$ for the transitions $2 \mathrm{p}^{3}{ }^{4} \mathrm{~S}_{3 / 2}^{\mathrm{o}}-2 \mathrm{~s} 2 \mathrm{p}^{2}{ }^{2} \mathrm{D}_{3 / 2}$ $(11-6), 2 \mathrm{p}^{3}{ }^{4} \mathrm{~S}_{3 / 2}^{\mathrm{o}}-2 \mathrm{~s} 2 \mathrm{p}^{2}{ }^{2} \mathrm{~S}_{1 / 2}(11-8)$ and $2 \mathrm{p}^{3}{ }^{2} \mathrm{D}_{3 / 2}^{\mathrm{o}}-2 \mathrm{~s} 2 \mathrm{p}^{2}$ ${ }^{4} \mathrm{P}_{5 / 2}(12-5)$, which are spin-forbidden transitions.

\subsection{Electron-ion scattering}

The collision strength can be defined as the measure of strength for a binary collision. It contains the information about this collision. It is a dimensionless quantity and is related to the collision cross section $\sigma_{i f}$ ( $i$ and $f$ are the initial and final levels, respectively) by the following relationship:

$$
\Omega_{i f}=\frac{k_{i}^{2} g_{i}}{\pi a_{0}^{2}} \sigma_{i f},
$$

where $k_{i}^{2}$ is the incident electron energy in Ryd and $g_{i}$ is the statistical weight of the initial level. We can say also that both collision strength and cross section describe the intrinsic probability of collisional excitation and de-excitation in an atomic transition at a particular electron energy.

We present in Table 5, collision strengths for several Ar XII lines at three electron energies $\varepsilon: 35,70$ and 105 Ryd and compare them to the results of Eissner et al. [21]. For Ar XIV, we present collision strengths at one electron energy $40 \mathrm{Ryd}$, and compare them with the calculations of [19]. The relative errors for Ar XII collision strengths are about 3\% for $\varepsilon=35$ Ryd, $9 \%$ for $\varepsilon=70$ Ryd and $16 \%$ for $\varepsilon=105$ Ryd. Our Ar XIV collision strengths agree well with those of [19] (about 4\%). 
Table 1: Present fine structure energy levels $E$ (in $\mathrm{cm}^{-1}$ ) for the Ar XII ion compared to the NIST values [38], to the experimental results (Edl) of Edlén [39], and to the results of Bhatia et al. [18] using the SST code with $l<8\left(\mathrm{SST}_{7}\right)$.

\begin{tabular}{|c|c|c|c|c|c|c|c|c|}
\hline$k$ & Level & $E$ & NIST & Edl & $\mathrm{SST}_{7}$ & $\Delta_{\mathrm{NIST}}$ & $\Delta_{\text {Edl }}$ & $\Delta_{\mathrm{SST}_{7}}$ \\
\hline 1 & $2 s^{2} 2 p^{3}{ }^{4} S^{\circ} 3 / 2$ & 0.0 & 0.0 & 0.0 & 0.0 & - & - & - \\
\hline 2 & $2 \mathrm{~s}^{2} 2 \mathrm{p}^{3}{ }^{2} \mathrm{D}^{\circ}{ }_{3 / 2}$ & 99057. & 94824. & 94815 & 98694 & 4.27 & 4.28 & 0.37 \\
\hline 3 & $2 \mathrm{~s}^{2} 2 \mathrm{p}^{3}{ }^{2} \mathrm{D}^{\circ}{ }_{5 / 2}$ & 102883. & 98155.7 & 98162 & 102577 & 4.59 & 4.59 & 0.30 \\
\hline 4 & $2 \mathrm{~s}^{2} 2 \mathrm{p}^{3}{ }^{2} \mathrm{P}_{1 / 2}^{\circ}$ & 149743. & 149186. & 149175 & 149188 & 0.37 & 0.38 & 0.37 \\
\hline 5 & $2 \mathrm{~s}^{2} 2 \mathrm{p}^{3}{ }^{2} \mathrm{P}_{3 / 2}^{\circ}$ & 155056. & 154061. & 154050 & 154551 & 0.64 & 0.65 & 0.33 \\
\hline 6 & $2 \mathrm{~s}\left({ }^{2} \mathrm{~S}\right) 2 \mathrm{p}^{4}\left({ }^{3} \mathrm{P}\right){ }^{4} \mathrm{P}_{5 / 2}$ & 446673. & 445931. & 445932 & 444555 & 0.17 & 0.17 & 0.47 \\
\hline 7 & $2 \mathrm{~s}\left({ }^{2} \mathrm{~S}\right) 2 \mathrm{p}^{4}\left({ }^{3} \mathrm{P}\right){ }^{4} \mathrm{P}_{3 / 2}$ & 458993. & 458106. & 458100 & 456653 & 0.19 & 0.19 & 0.51 \\
\hline 8 & $2 s\left({ }^{2} S\right) 2 p^{4}\left({ }^{3} P\right){ }^{4} P_{1 / 2}$ & 465310. & 464058. & 464043 & 462868 & 0.27 & 0.27 & 0.52 \\
\hline 9 & $2 \mathrm{~s}\left({ }^{2} \mathrm{~S}\right) 2 \mathrm{p}^{4}\left({ }^{1} \mathrm{D}\right){ }^{2} \mathrm{D}_{3 / 2}$ & 626680. & 613833. & 613803 & 623875 & 2.05 & 2.05 & 0.45 \\
\hline 10 & $2 \mathrm{~s}\left({ }^{2} \mathrm{~S}\right) 2 \mathrm{p}^{4}\left({ }^{1} \mathrm{D}\right){ }^{2} \mathrm{D}_{5 / 2}$ & 627743. & 614466. & 614431 & 624902 & 2.11 & 2.12 & 0.45 \\
\hline 11 & $2 s\left({ }^{2} S\right) 2 p^{4}\left({ }^{1} S\right){ }^{2} S_{1 / 2}$ & 728384. & 715379. & 715277 & 724882 & 1.79 & 1.80 & 0.48 \\
\hline 12 & $2 \mathrm{~s}\left({ }^{2} \mathrm{~S}\right) 2 \mathrm{p}^{4}\left({ }^{3} \mathrm{P}\right){ }^{2} \mathrm{P}_{3 / 2}$ & 767374 . & 745762 . & 745738 & 764830 & 2.82 & 2.82 & 0.33 \\
\hline 13 & $2 \mathrm{~s}\left({ }^{2} \mathrm{~S}\right) 2 \mathrm{p}^{4}\left({ }^{3} \mathrm{P}\right){ }^{2} \mathrm{P}_{1 / 2}$ & 783733. & 761838. & 761797 & 780830 & 2.79 & 2.80 & 0.37 \\
\hline 14 & $2 \mathrm{p}^{5}{ }^{2} \mathrm{P}_{3 / 2}^{\circ}$ & 1203351. & 1166700. & 1166585 & 1198170 & 3.05 & 3.06 & 0.43 \\
\hline 15 & $2 \mathrm{p}^{5}{ }^{2} \mathrm{P}^{\circ}{ }_{1 / 2}$ & 1224440. & 1186244. & 1186193 & 1218881 & 3.12 & 3.12 & 0.45 \\
\hline 16 & $2 \mathrm{~s}^{2} 2 \mathrm{p}^{2}\left({ }^{3} \mathrm{P}\right) 3 \mathrm{~s}{ }^{4} \mathrm{P}_{1 / 2}$ & 2877392. & 2867000. & - & 2882081 & 0.36 & - & 0.16 \\
\hline 17 & $2 s^{2} 2 p^{2}\left({ }^{3} P\right) 3 s{ }^{4} P_{3 / 2}$ & 2885722. & 2875200. & - & 2890248 & 0.36 & - & 0.16 \\
\hline 18 & $2 \mathrm{~s}^{2} 2 \mathrm{p}^{2}\left({ }^{3} \mathrm{P}\right) 3 \mathrm{~s}^{4} \mathrm{P}_{5 / 2}$ & 2897052. & 2883920. & - & 2901387 & 0.45 & - & 0.15 \\
\hline 19 & $2 \mathrm{~s}^{2} 2 \mathrm{p}^{2}\left({ }^{3} \mathrm{P}\right) 3 \mathrm{~s}^{2} \mathrm{P}_{1 / 2}$ & 2909658. & - & - & 2914248 & - & - & 0.16 \\
\hline 20 & $2 \mathrm{~s}^{2} 2 \mathrm{p}^{2}\left({ }^{3} \mathrm{P}\right) 3 \mathrm{~s}^{2} \mathrm{P}_{3 / 2}$ & 2922856. & 2912070. & - & 2927228 & 0.37 & - & 0.15 \\
\hline 21 & $2 s^{2} 2 p^{2}\left({ }^{1} D\right) 3 s^{2} D_{5 / 2}$ & 2969949. & 2956650. & - & 2973839 & 0.45 & - & 0.13 \\
\hline 22 & $2 s^{2} 2 p^{2}\left({ }^{1} D\right) 3 s^{2} D_{3 / 2}$ & 2970712. & 2958030. & - & 2974586 & 0.43 & - & 0.13 \\
\hline 23 & $2 s^{2} 2 p^{2}\left({ }^{3} P\right) 3 p{ }^{4} D_{1 / 2}$ & 2997407. & - & - & 3001934 & - & - & 0.15 \\
\hline 24 & $2 s^{2} 2 p^{2}\left({ }^{3} P\right) 3 p^{2} S_{1 / 2}$ & 3003227. & - & - & 3007678 & - & - & 0.15 \\
\hline 25 & $2 \mathrm{~s}^{2} 2 \mathrm{p}^{2}\left({ }^{3} \mathrm{P}\right) 3 \mathrm{p}^{4} \mathrm{D}^{\circ}{ }_{3 / 2}$ & 3006437. & 2996300. & - & 3010901 & 0.34 & - & 0.34 \\
\hline 26 & $2 s^{2} 2 p^{2}\left({ }^{3} P\right) 3 p{ }^{4} D_{5 / 2}$ & 3014426. & - & - & 3018750 & - & - & 0.14 \\
\hline 27 & $2 s^{2} 2 p^{2}\left({ }^{3} \mathrm{P}\right) 3 \mathrm{p}^{4} \mathrm{P}^{\circ}{ }_{1 / 2}$ & 3021348. & 3010700. & - & 3025651 & 0.35 & - & 0.14 \\
\hline 28 & $2 \mathrm{~s}^{2} 2 \mathrm{p}^{2}\left({ }^{3} \mathrm{P}\right) 3 \mathrm{p}^{4} \mathrm{P}_{3 / 2}^{\circ}$ & 3022117. & 3011700 . & - & 3026450 & 0.34 & - & 0.14 \\
\hline 29 & $2 \mathrm{~s}^{2} 2 \mathrm{p}^{2}\left({ }^{3} \mathrm{P}\right) 3 \mathrm{p}^{4} \mathrm{D}^{\circ}{ }_{7 / 2}$ & 3024837. & 3011470 . & - & 3028996 & 0.44 & - & 0.14 \\
\hline 30 & $2 \mathrm{~s}^{2} 2 \mathrm{p}^{2}\left({ }^{3} \mathrm{P}\right) 3 \mathrm{p}^{4} \mathrm{P}^{\circ}{ }_{5 / 2}$ & 3028695 . & - & - & 3032908 & - & - & 0.14 \\
\hline 31 & $2 s^{2} 2 p^{2}\left({ }^{3} P\right) 3 p^{2} D_{3 / 2}$ & 3037364. & - & - & 3041562 & - & - & 0.14 \\
\hline 32 & $2 \mathrm{~s}^{2} 2 \mathrm{p}^{2}\left({ }^{3} \mathrm{P}\right) 3 \mathrm{p}^{2} \mathrm{D}^{\circ}{ }_{5 / 2}$ & 3051869. & - & - & 3055790 & & - & 0.13 \\
\hline 33 & $2 \mathrm{~s}^{2} 2 \mathrm{p}^{2}\left({ }^{3} \mathrm{P}\right) 3 \mathrm{p}^{2} \mathrm{P}^{\circ}{ }_{3 / 2}$ & 3062921. & - & - & 3066868 & - & - & 0.13 \\
\hline 34 & $2 s^{2} 2 p^{2}\left({ }^{3} \mathrm{P}\right) 3 \mathrm{p}^{2} \mathrm{P}_{1 / 2}^{\circ}$ & 3064996. & - & - & 3068888 & - & - & 0.13 \\
\hline 35 & $2 s^{2} 2 p^{2}\left({ }^{3} P\right) 3 p^{4} S^{\circ}{ }_{3 / 2}$ & 3069689. & 3059000 . & - & 3074109 & 0.35 & - & 0.14 \\
\hline 36 & $2 s^{2} 2 p^{2}\left({ }^{1} S\right) 3 s^{2} S_{1 / 2}$ & 3072782. & - & - & 3075853 & - & - & 0.10 \\
\hline 37 & $2 \mathrm{~s}^{2} 2 \mathrm{p}^{2}\left({ }^{1} \mathrm{D}\right) 3 \mathrm{p}^{2} \mathrm{~F}^{\circ}{ }_{5 / 2}$ & 3097254 . & - & - & 3100983 & - & - & 0.12 \\
\hline 38 & $2 \mathrm{~s}^{2} 2 \mathrm{p}^{2}\left({ }^{1} \mathrm{D}\right) 3 \mathrm{p}^{2} \mathrm{~F}^{\circ} 7 / 2$ & 3100806. & - & - & 3104508 & - & - & 0.12 \\
\hline 39 & $2 \mathrm{~s}^{2} 2 \mathrm{p}^{2}\left({ }^{1} \mathrm{D}\right) 3 \mathrm{p}^{2} \mathrm{D}^{\circ}{ }_{3 / 2}$ & 3133354. & - & - & 3137108 & - & - & 0.12 \\
\hline 40 & $2 \mathrm{~s}^{2} 2 \mathrm{p}^{2}\left({ }^{1} \mathrm{D}\right) 3 \mathrm{p}^{2} \mathrm{D}^{\circ}{ }_{5 / 2}$ & 3134758. & 3124840 . & - & 3138442 & 0.32 & - & 0.12 \\
\hline 41 & $2 \mathrm{~s}^{2} 2 \mathrm{p}^{2}\left({ }^{1} \mathrm{D}\right) 3 \mathrm{p}^{2} \mathrm{P}^{\circ}{ }_{1 / 2}$ & 3144705 . & 3133800 . & - & 3148104 & 0.35 & - & 0.11 \\
\hline 42 & $2 s^{2} 2 p^{2}\left({ }^{3} P\right) 3 d^{4} F_{3 / 2}$ & 3152201. & - & - & 3158121 & - & - & 0.19 \\
\hline 43 & $2 \mathrm{~s}^{2} 2 \mathrm{p}^{2}\left({ }^{1} \mathrm{D}\right) 3 \mathrm{p}^{2} \mathrm{P}_{3 / 2}^{\circ}$ & 3155822 . & 3142510 . & - & 3158989 & 0.42 & - & 0.10 \\
\hline 44 & $2 s^{2} 2 p^{2}\left({ }^{3} P\right) 3 d^{4} F_{5 / 2}$ & 3156342. & - & - & 3162167 & - & - & 0.18 \\
\hline 45 & $2 s^{2} 2 p^{2}\left({ }^{3} P\right) 3 d^{4} F_{7 / 2}$ & 3162712 . & - & - & 3168409 & - & - & 0.18 \\
\hline 46 & $2 s^{2} 2 p^{2}\left({ }^{3} P\right) 3 d^{4} D_{3 / 2}$ & 3170960 . & - & - & 3176405 & - & - & 0.17 \\
\hline 47 & $2 s^{2} 2 p^{2}\left({ }^{3} P\right) 3 d^{4} F_{9 / 2}$ & 3171344. & - & - & 3176890 & - & - & 0.17 \\
\hline 48 & $2 \mathrm{~s}^{2} 2 \mathrm{p}^{2}\left({ }^{3} \mathrm{P}\right) 3 \mathrm{~d}^{4} \mathrm{D}_{1 / 2}$ & 3172100 . & - & - & 3177624 & - & - & 0.17 \\
\hline 49 & $2 \mathrm{~s}^{2} 2 \mathrm{p}^{2}\left({ }^{3} \mathrm{P}\right) 3 \mathrm{~d}^{4} \mathrm{D}_{5 / 2}$ & 3175790 & - & - & 3181224 & - & - & 0.17 \\
\hline 50 & $2 s^{2} 2 p^{2}\left({ }^{3} P\right) 3 d^{2} P_{3 / 2}$ & 3179808. & 3276700 . & - & 3185054 & 3.05 & - & 0.16 \\
\hline 51 & $2 \mathrm{~s}^{2} 2 \mathrm{p}^{2}\left({ }^{3} \mathrm{P}\right) 3 \mathrm{~d}^{4} \mathrm{D}_{7 / 2}$ & 3180482 . & - & - & 3185910 & - & - & 0.17 \\
\hline 52 & $2 s^{2} 2 p^{2}\left({ }^{3} P\right) 3 d^{2} F_{5 / 2}$ & 3187457. & 3175950 & - & 3192699 & 0.36 & - & 0.16 \\
\hline
\end{tabular}




\begin{tabular}{|c|c|c|c|c|c|c|}
\hline \multicolumn{7}{|c|}{ Table 1: Continued. } \\
\hline$k$ & Level & $E$ & NIST & $\mathrm{SST}_{7}$ & $\Delta_{\mathrm{NIST}}$ & $\Delta_{\mathrm{SST}_{7}}$ \\
\hline 53 & $2 s^{2} 2 p^{2}\left({ }^{3} P\right) 3 d^{2} P_{1 / 2}$ & 3190465. & 3271350 . & 3195372 & 2.54 & 0.15 \\
\hline 54 & $2 s^{2} 2 p^{2}\left({ }^{3} P\right) 3 d^{4} P_{5 / 2}$ & 3198865. & 3187350. & 3203320 & 0.36 & 0.14 \\
\hline 55 & $2 s^{2} 2 p^{2}\left({ }^{3} \mathrm{P}\right) 3 d^{2} \mathrm{~F}_{7 / 2}$ & 3202525 . & 3189920. & 3207518 & 0.39 & 0.16 \\
\hline 56 & $2 s^{2} 2 p^{2}\left({ }^{3} P\right) 3 d^{4} P_{3 / 2}$ & 3203183. & 3190100. & 3207531 & 0.41 & 0.14 \\
\hline 57 & $2 s^{2} 2 p^{2}\left({ }^{3} P\right) 3 d^{4} P_{1 / 2}$ & 3205386. & - & 3209682 & - & 0.13 \\
\hline 58 & $2 \mathrm{~s}^{2} 2 \mathrm{p}^{2}\left({ }^{1} \mathrm{~S}\right) 3 \mathrm{p}^{2} \mathrm{P}^{\circ}{ }_{1 / 2}$ & 3216498. & - & 3219498 & - & 0.09 \\
\hline 59 & $2 \mathrm{~s}^{2} 2 \mathrm{p}^{2}\left({ }^{1} \mathrm{~S}\right) 3 \mathrm{p}^{2} \mathrm{P}^{\circ}$ & 3217330. & _- & 3220280 & _- & 0.09 \\
\hline 60 & $2 \mathrm{~s}^{2} 2 \mathrm{p}^{2}\left({ }^{3} \mathrm{P}\right) 3 \mathrm{~d}^{2} \mathrm{D}_{3 / 2}$ & 3238702 . & 3221580 . & 3242310 & 0.53 & 0.11 \\
\hline 61 & $2 s^{2} 2 p^{2}\left({ }^{3} \mathrm{P}\right) 3 d^{2} D_{5 / 2}$ & 3240595 . & 3226750 . & 3244179 & 0.43 & 0.11 \\
\hline 62 & $2 \mathrm{~s}^{2} 2 \mathrm{p}^{2}\left({ }^{1} \mathrm{D}\right) 3 \mathrm{~d}^{2} \mathrm{G}_{7 / 2}$ & 3251209. & 3258510 . & 3255926 & 0.22 & 0.15 \\
\hline 63 & $2 \mathrm{~s}^{2} 2 \mathrm{p}^{2}\left({ }^{1} \mathrm{D}\right) 3 \mathrm{~d}^{2} \mathrm{G}_{9 / 2}$ & 3253192. & - & 3257851 & - & 0.14 \\
\hline 64 & $2 \mathrm{~s}^{2} 2 \mathrm{p}^{2}\left({ }^{1} \mathrm{D}\right) 3 \mathrm{~d}^{2} \mathrm{D}_{3 / 2}$ & 3268759 . & - & 3272678 & - & 0.12 \\
\hline 65 & $2 \mathrm{~s}^{2} 2 \mathrm{p}^{2}\left({ }^{1} \mathrm{D}\right) 3 \mathrm{~d}^{2} \mathrm{D}_{5 / 2}$ & 3271306. & - & 3274957 & - & 0.11 \\
\hline 66 & $2 \mathrm{~s}^{2} 2 \mathrm{p}^{2}\left({ }^{1} \mathrm{D}\right) 3 \mathrm{~d}^{2} \mathrm{~F}_{7 / 2}$ & 3276720 . & - & 3279841 & - & 0.10 \\
\hline 67 & $2 s^{2} 2 p^{2}\left({ }^{1} D\right) 3 d^{2} F_{5 / 2}$ & 3281986. & 3264720 . & 3285089 & 0.53 & 0.09 \\
\hline 68 & $2 s^{2} 2 p^{2}\left({ }^{1} D\right) 3 d^{2} P_{1 / 2}$ & 3285581. & 3271350. & 3289124 & 0.43 & 0.11 \\
\hline 69 & $2 s^{2} 2 p^{2}\left({ }^{1} D\right) 3 d^{2} P_{3 / 2}$ & 3291020. & 3276700 . & 3294359 & 0.43 & 0.10 \\
\hline 70 & $2 s^{2} 2 p^{2}\left({ }^{1} D\right) 3 d^{2} S_{1 / 2}$ & 3297762. & - & 3301242 & - & 0.11 \\
\hline 71 & $2 \mathrm{~s}^{2} 2 \mathrm{p}^{2}\left({ }^{1} \mathrm{~S}\right) 3 \mathrm{~d}^{2} \mathrm{D}_{5 / 2}$ & 3368328. & 3226750 . & 3371651 & 4.20 & 0.10 \\
\hline 72 & $2 \mathrm{~s}^{2} 2 \mathrm{p}^{2}\left({ }^{1} \mathrm{~S}\right) 3 \mathrm{~d}^{2} \mathrm{D}_{3 / 2}$ & 3371236. & 3221580 . & 3374427 & 4.43 & 0.09 \\
\hline
\end{tabular}

The comparisons performed for our atomic and collision data show an acceptable overall agreement between the experimental and the other theoretical results. As it has been mentioned before, these parameters represent intermediate input data for our line broadening calculations, and their comparisons are very important for the evaluation of the accuracy of line broadening results. The good agreement found can indicate that we can trust the intermediate data used in the calculations of Stark broadening.

\subsection{Line broadening results}

We perform Stark broadening calculations for $12 \mathrm{Ar}$ XII and 12 Ar XIV lines. Ar XII calculations are shown in Table 6 and the results of Ar XIV are shown in Table 7. Results are presented for the range of electron temperature form $5 \times 10^{5}$ to $5 \times 10^{6} \mathrm{~K}$ and at electron density $N_{e}=10^{18} \mathrm{~cm}^{-3}$. Quantum results $W$ are calculated using the expression (1) using 6 configurations for Ar XII and 8 configurations for Ar XIV. There are no other Stark widths results for all these lines to compare with. The acceptable agreements found between our atomic and collision parameters and other results show that our line broadening data are calculated with an acceptable accuracy. Measurements or new calculations of Ar XII and Ar XIV line broadening would be very helpful to confirm our conclusions.

\section{Conclusions}

We have calculated in the present paper quantum mechanical Stark widths for $12 \mathrm{Ar}$ XII and $12 \mathrm{Ar}$ XIV lines for the range of electron temperature from $5 \times 10^{5}$ to $5 \times 10^{6} \mathrm{~K}$ and at electron density $N_{e}=10^{18} \mathrm{~cm}^{-3}$. We have used our quantum method developed in Elabidi et al. $[25,26]$ together with the UCL packages (SST/DW/JAJOM). Radiative and collision parameters have been also calculated and compared to other results, and good agreement has been found. This can give an idea about the accuracy of our line broadening calculation. To the best of our knowledge, no other results for the Stark broadening have been found to perform comparisons. New theoretical or experimental evaluations of line widths for these ions maybe interesting for checking our calculations.

\section{Acknowledgments}

This work has been supported by the Tunisian Laboratory of Molecular Spectroscopy and Dynamics LR18ES02 and the French Laboratory LERMA of the Paris Observatory and CNRS UMR 8112. One of us (S.S.B) acknowledges also financial support from the Programme National de Physique Stellaire of CNRS/INSU, CEA, and CNES, France.

\section{References}

[1] Rauch T, Ziegler M, Werner K, Kruk J W, Oliveira C M, Putte D Vande, Mignani R P, Kerber F. High-resolution FUSE and HST ultraviolet spectroscopy of the white dwarf central star of Sh 2-216. A\&A 2007;470:317329. doi:10.1051/0004-6361:20077166.

[2] Werner K, Rauch T, Kruk J W. Discovery of photospheric argon in very hot central stars of planetary nebulae and white dwarfs. A\&A 2007;466:317-322. doi: 10.1051/0004-6361:20077101. 
Table 2: Our fine structure energy levels $E$ (in $\mathrm{cm}^{-1}$ ) for the Ar XIV ion compared to those of NIST [38], to those of Liang et al. [40] (AS) obtained using the code AUTOSTRUCTURE [41] and to those of Aggarwal et al. [42] using the GRASP code [43] (GRASP).

\begin{tabular}{|c|c|c|c|c|c|c|c|c|}
\hline$k$ & Level & $E$ & NIST & AS & GRASP & $\Delta_{\text {NIST }}$ & $\Delta_{\mathrm{AS}}$ & $\Delta_{\text {GRASP }}$ \\
\hline 1 & $2 s^{2} 2 p^{2} \mathrm{P}^{\circ}{ }_{1 / 2}$ & 0.0 & 0.0 & 0.0 & 0.0 & - & - & - \\
\hline 2 & $2 \mathrm{~s}^{2} 2 \mathrm{p}^{2} \mathrm{P}_{3 / 2}^{\circ}$ & 22821. & 22656. & 22408.36 & 22551.10 & 0.72 & 1.81 & 1.18 \\
\hline 3 & $2 \mathrm{~s} 2 \mathrm{p}^{2}{ }^{4} \mathrm{P}_{1 / 2}$ & 223345 . & 230296. & 224818.81 & 226081.61 & 3.11 & 0.66 & 1.23 \\
\hline 4 & $2 \mathrm{~s} 2 \mathrm{p}^{2}{ }^{4} \mathrm{P}_{3 / 2}$ & 232122 . & 238954. & 233400.26 & 234608.23 & 2.94 & 0.55 & 1.07 \\
\hline 5 & $2 \mathrm{~s} 2 \mathrm{p}^{2}{ }^{4} \mathrm{P}_{5 / 2}$ & 244681. & 250423. & 245701.81 & 246053.87 & 2.35 & 0.42 & 0.56 \\
\hline 6 & $2 \mathrm{~s} 2 \mathrm{p}^{2}{ }^{2} \mathrm{D}_{3 / 2}$ & 415525. & 410254. & 413610.86 & 414786.56 & 1.27 & 0.46 & 0.18 \\
\hline 7 & $2 \mathrm{~s} 2 \mathrm{p}^{2}{ }^{2} \mathrm{D}_{5 / 2}$ & 416843. & 411205. & 414905.76 & 415675.43 & 1.35 & 0.46 & 0.28 \\
\hline 8 & $2 \mathrm{~s} 2 \mathrm{p}^{2}{ }^{2} \mathrm{~S}_{1 / 2}$ & 519855. & 514401. & 519298.85 & 522175.87 & 1.05 & 0.11 & 0.45 \\
\hline 9 & $2 \mathrm{~s} 2 \mathrm{p}^{2}{ }^{2} \mathrm{P}_{1 / 2}$ & 557136. & 545244. & 551539.67 & 554054.67 & 2.13 & 1.00 & 0.55 \\
\hline 10 & $2 \mathrm{~s} 2 \mathrm{p}^{2}{ }^{2} \mathrm{P}_{3 / 2}$ & 568721. & 554678. & 561986.67 & 563854.25 & 2.47 & 1.18 & 0.86 \\
\hline 11 & $2 \mathrm{p}^{3}{ }^{4} \mathrm{~S}_{3 / 2}^{\circ}$ & 716725 . & 718925. & 717780.71 & 719341.60 & 0.30 & 0.15 & 0.37 \\
\hline 12 & $2 \mathrm{p}^{32} \mathrm{D}^{\circ}{ }_{3 / 2}$ & 822104. & 810387. & 817586.78 & 818917.59 & 1.42 & 0.55 & 0.39 \\
\hline 13 & $2 \mathrm{p}^{3}{ }^{2} \mathrm{D}^{\circ}{ }_{5 / 2}$ & 824973. & 812956. & 820330.21 & 820673.39 & 1.46 & 0.56 & 0.52 \\
\hline 14 & $2 \mathrm{p}^{32} \mathrm{P}^{\circ}{ }_{1 / 2}$ & 921826. & 908793. & 918435.36 & 920501.78 & 1.41 & 0.37 & 0.14 \\
\hline 15 & $2 \mathrm{p}^{3}{ }^{2} \mathrm{P}_{3 / 2}^{\circ}$ & 926564. & 913056. & 923077.25 & 924638.89 & 1.46 & 0.38 & 0.21 \\
\hline 16 & $2 s^{2} 3 s^{2} S_{1 / 2}$ & 3452445 . & - & 3417360. & 3410220. & - & 1.02 & 1.22 \\
\hline 17 & $2 s^{2} 3 p^{2} \mathrm{P}_{1 / 2}^{\circ}$ & 3570636 . & 3533890. & 3530740 . & 3524080 . & 1.03 & 1.12 & 1.30 \\
\hline 18 & $2 s^{2} 3 p^{2} \mathrm{P}_{3 / 2}^{\circ}$ & 3576283. & 3534840 . & 3537000 . & 3530310 . & 1.16 & 1.10 & 1.29 \\
\hline 19 & $2 \mathrm{~s} 2 \mathrm{p} 3 \mathrm{~s}{ }^{4} \mathrm{P}^{\circ}{ }_{1 / 2}$ & 3651843. & - & 3649360. & 3646140. & - & 0.07 & 0.16 \\
\hline 20 & $2 \mathrm{~s} 2 \mathrm{p} 3 \mathrm{~s}{ }^{4} \mathrm{P}_{3 / 2}^{\circ}$ & 3659152. & - & 3656480 . & 3653220. & - & 0.07 & 0.16 \\
\hline 21 & $2 \mathrm{~s} 2 \mathrm{p} 3 \mathrm{~s}{ }^{4} \mathrm{P}^{\circ}{ }_{5 / 2}$ & 3672972 . & - & 3670260 . & 3667470 . & - & 0.07 & 0.15 \\
\hline 22 & $2 s^{2} 3 d^{2} D_{3 / 2}$ & 3682658. & 3640470 . & 3644120 . & 3636450 . & 1.15 & 1.05 & 1.25 \\
\hline 23 & $2 s^{2} 3 d^{2} D_{5 / 2}$ & 3684537. & 3641780 . & 3646022 . & 3638180 . & 1.16 & 1.05 & 1.26 \\
\hline 24 & $2 \mathrm{~s} 2 \mathrm{p}\left({ }^{3} \mathrm{P}^{\circ}\right) 3 \mathrm{~s}{ }^{2} \mathrm{P}^{\circ}{ }_{1 / 2}$ & 3716193. & 3705800 . & 3707520. & 3705890. & 0.28 & 0.23 & 0.28 \\
\hline 25 & $2 \mathrm{~s} 2 \mathrm{p}\left({ }^{3} \mathrm{P}^{\circ}\right) 3 \mathrm{~s}^{2} \mathrm{P}^{\circ}{ }_{3 / 2}$ & 3731468. & 3720820 . & 3722740 . & 3721360 . & 0.29 & 0.23 & 0.27 \\
\hline 26 & $2 \mathrm{~s} 2 \mathrm{p} 3 \mathrm{p}^{4} \mathrm{D}_{1 / 2}$ & 3751754. & - & 3750890. & 3746090. & - & 0.02 & 0.15 \\
\hline 27 & $2 \mathrm{~s} 2 \mathrm{p} 3 \mathrm{p}^{4} \mathrm{D}_{3 / 2}$ & 3757322 . & - & 3757280 . & 3752320 . & - & $1 \mathrm{E}-4$ & 0.13 \\
\hline 28 & $2 \mathrm{~s} 2 \mathrm{p} 3 \mathrm{p}^{4} \mathrm{D}_{5 / 2}$ & 3766442. & - & 3767910. & 3763070. & - & 0.04 & 0.09 \\
\hline 29 & $2 \mathrm{~s} 2 \mathrm{p}\left({ }^{3} \mathrm{P}^{\circ}\right) 3 \mathrm{p}^{2} \mathrm{P}_{3 / 2}$ & 3775532. & - & 3766880. & 3762740. & - & 0.23 & 0.34 \\
\hline 30 & $2 \mathrm{~s} 2 \mathrm{p}\left({ }^{3} \mathrm{P}^{\circ}\right) 3 \mathrm{p}^{2} \mathrm{P}_{1 / 2}$ & 3777347. & - & 3764540. & 3760380. & - & 0.34 & 0.45 \\
\hline 31 & $2 \mathrm{~s} 2 \mathrm{p} 3 \mathrm{p}^{4} \mathrm{D}_{7 / 2}$ & 3778746. & - & 3780130. & 3775700 . & - & 0.04 & 0.08 \\
\hline 32 & $2 \mathrm{~s} 2 \mathrm{p} 3 \mathrm{p}^{4} \mathrm{~S}_{3 / 2}$ & 3789084. & - & 3789170. & 3784860 . & - & 0.00 & 0.11 \\
\hline 33 & $2 \mathrm{~s} 2 \mathrm{p} 3 \mathrm{p}^{4} \mathrm{P}_{1 / 2}$ & 3799410. & - & 3796720. & 3796940. & - & 0.07 & 0.06 \\
\hline 34 & $2 \mathrm{~s} 2 \mathrm{p} 3 \mathrm{p}^{4} \mathrm{P}_{3 / 2}$ & 3807087. & - & 3805110. & 3804980 . & - & 0.05 & 0.06 \\
\hline 35 & $2 \mathrm{~s} 2 \mathrm{p} 3 \mathrm{p}^{4} \mathrm{P}_{5 / 2}$ & 3812981. & - & 3810490. & 3810380. & - & 0.07 & 0,07 \\
\hline 36 & $2 \mathrm{~s} 2 \mathrm{p}\left({ }^{3} \mathrm{P}^{\circ}\right) 3 \mathrm{p}{ }^{2} \mathrm{D}_{3 / 2}$ & 3822328. & - & 3816280 . & 3814330. & - & 0.16 & 0.21 \\
\hline 37 & $2 \mathrm{~s} 2 \mathrm{p}\left({ }^{3} \mathrm{P}^{\circ}\right) 3 \mathrm{p}^{2} \mathrm{D}_{5 / 2}$ & 3835998. & 3828850 . & 3829960. & 3828830 . & 0.19 & 0.16 & 0.19 \\
\hline 38 & $2 \mathrm{~s} 2 \mathrm{p}\left({ }^{3} \mathrm{P}^{\circ}\right) 3 \mathrm{p}^{2} \mathrm{~S}_{1 / 2}$ & 3863078. & - & 3859670 . & 3858310 . & - & 0.09 & 0.12 \\
\hline 39 & $2 \mathrm{~s} 2 \mathrm{p}\left({ }^{1} \mathrm{P}^{\circ}\right) 3 \mathrm{~s}^{2} \mathrm{P}^{\circ}{ }_{1 / 2}$ & 3904496. & - & 3887130. & 3884950 . & - & 0.44 & 0.50 \\
\hline 40 & $2 \mathrm{~s} 2 \mathrm{p}\left({ }^{1} \mathrm{P}^{\circ}\right) 3 \mathrm{~s}^{2} \mathrm{P}^{\circ}{ }_{3 / 2}$ & 3905759. & - & 3887480 . & 3889710. & - & 0.47 & 0.41 \\
\hline 41 & $2 \mathrm{~s} 2 \mathrm{p}\left({ }^{1} \mathrm{P}^{\circ}\right) 3 \mathrm{p}^{2} \mathrm{D}_{3 / 2}$ & 4012579. & - & - & 4000850. & - & - & 0.29 \\
\hline 42 & $2 \mathrm{~s} 2 \mathrm{p}\left({ }^{1} \mathrm{P}^{\circ}\right) 3 \mathrm{p}^{2} \mathrm{D}_{5 / 2}$ & 4015738. & - & - & 4004080. & - & - & 0.29 \\
\hline 43 & $2 \mathrm{~s} 2 \mathrm{p}\left({ }^{1} \mathrm{P}^{\circ}\right) 3 \mathrm{p}^{2} \mathrm{P}_{1 / 2}$ & 4017499. & - & - & 3998960. & - & - & 0.46 \\
\hline 44 & $2 \mathrm{~s} 2 \mathrm{p}\left({ }^{1} \mathrm{P}^{\circ}\right) 3 \mathrm{p}^{2} \mathrm{P}_{3 / 2}$ & 4023117. & - & - & 4005390. & - & - & 0.44 \\
\hline 45 & $2 \mathrm{~s} 2 \mathrm{p}\left({ }^{1} \mathrm{P}^{\circ}\right) 3 \mathrm{p}^{2} \mathrm{~S}_{1 / 2}$ & 4051416. & - & - & 4031960. & - & - & 0.48 \\
\hline
\end{tabular}


Table 3: Present Ar XII radiative decay rates $A_{i j}$ and weighted oscillator strengths $g f$ are compared to those of Zhang and Sampson [20]: ZS99, to those of Merkelis et al. [44]: M97 and to those of Bhatia et al. [18] using the SST code with $l<8$ : $\mathrm{SST}_{7}$. Our line strengths $S$ are also presented. $i$ and $j$ label the levels of Table 1 .

\begin{tabular}{|c|c|c|c|c|c|c|c|c|}
\hline Levels & & $A_{i j}($ & & & & $g f$ & & $S$ \\
\hline$i-j$ & Present & ZS99 & M97 & $\mathrm{SST}_{7}$ & Present & M97 & $\mathrm{SST}_{7}$ & \\
\hline $6-1$ & $6.726 \mathrm{E}+09$ & $7.265 \mathrm{E}+09$ & $6.106 \mathrm{E}+09$ & $6.7 \mathrm{E}+09$ & $3.032 \mathrm{E}-01$ & $2.780 \mathrm{E}-01$ & $3.060 \mathrm{E}-01$ & 0.223502 \\
\hline $6-2$ & $8.061 \mathrm{E}+06$ & $7.835 \mathrm{E}+06$ & $6.693 \mathrm{E}+06$ & - & $6.001 \mathrm{E}-04$ & $4.920 \mathrm{E}-04$ & - & 0.000568 \\
\hline $6-3$ & $1.383 \mathrm{E}+07$ & - & - & - & $1.053 \mathrm{E}-03$ & $9.480 \mathrm{E}-04$ & - & 0.001008 \\
\hline $6-5$ & $5.039 \mathrm{E}+06$ & - & - & - & $5.330 \mathrm{E}-04$ & $4.520 \mathrm{E}-04$ & - & 0.000602 \\
\hline $7-1$ & $7.315 \mathrm{E}+09$ & - & - & $7.3 \mathrm{E}+09$ & $2.082 \mathrm{E}-01$ & $1.910 \mathrm{E}-01$ & $2.100 \mathrm{E}-01$ & 0.149350 \\
\hline $7-3$ & $1.115 \mathrm{E}+06$ & $8.745 \mathrm{E}+05$ & $1.074 \mathrm{E}+06$ & - & $5.275 \mathrm{E}-05$ & $5.010 \mathrm{E}-05$ & - & 0.000049 \\
\hline $7-5$ & $1.257 \mathrm{E}+07$ & - & - & - & $8.163 \mathrm{E}-04$ & $6.740 \mathrm{E}-04$ & - & 0.000884 \\
\hline $8-1$ & $7.676 \mathrm{E}+09$ & - & - & $7.6 \mathrm{E}+09$ & $1.063 \mathrm{E}-01$ & $9.720 \mathrm{E}-02$ & $1.070 \mathrm{E}-01$ & 0.075210 \\
\hline $8-2$ & $3.576 \mathrm{E}+06$ & - & - & - & $7.993 \mathrm{E}-05$ & $7.120 \mathrm{E}-05$ & - & 0.000072 \\
\hline $8-4$ & $5.886 \mathrm{E}+06$ & - & - & - & $1.772 \mathrm{E}-04$ & $1.470 \mathrm{E}-04$ & - & 0.000185 \\
\hline $9-1$ & $7.014 \mathrm{E}+06$ & - & - & - & $1.071 \mathrm{E}-04$ & $8.870 \mathrm{E}-05$ & - & 0.000056 \\
\hline $9-2$ & $1.697 \mathrm{E}+10$ & $1.754 \mathrm{E}+10$ & $1.487 \mathrm{E}+10$ & $1.7 \mathrm{E}+10$ & $3.655 \mathrm{E}-01$ & $3.340 \mathrm{E}-01$ & $3.680 \mathrm{E}-01$ & 0.228078 \\
\hline $9-3$ & $9.149 \mathrm{E}+08$ & - & - & $9.3 \mathrm{E}+08$ & $2.000 \mathrm{E}-02$ & $1.850 \mathrm{E}-02$ & $2.040 \mathrm{E}-02$ & 0.012569 \\
\hline $9-4$ & $1.849 \mathrm{E}+09$ & $2.007 \mathrm{E}+09$ & $1.675 \mathrm{E}+09$ & $1.8 \mathrm{E}+09$ & $4.875 \mathrm{E}-02$ & $4.690 \mathrm{E}-02$ & $4.930 \mathrm{E}-02$ & 0.033650 \\
\hline $9-5$ & $4.988 \mathrm{E}+07$ & - & - & - & $1.345 \mathrm{E}-03$ & $2.680 \mathrm{E}-04$ & - & 0.000939 \\
\hline $10-1$ & $1.029 \mathrm{E}+06$ & $1.646 \mathrm{E}+05$ & $6.204 \mathrm{E}+05$ & - & $2.348 \mathrm{E}-05$ & $1.490 \mathrm{E}-05$ & - & 0.000012 \\
\hline $10-2$ & $2.340 \mathrm{E}+08$ & - & - & - & $7.531 \mathrm{E}-03$ & $8.020 \mathrm{E}-03$ & - & 0.004689 \\
\hline $10-3$ & $1.559 \mathrm{E}+10$ & $1.615 \mathrm{E}+10$ & $1.357 \mathrm{E}+10$ & $1.6 \mathrm{E}+10$ & $5.091 \mathrm{E}-01$ & $4.620 \mathrm{E}-01$ & $5.130 \mathrm{E}-01$ & 0.319306 \\
\hline $10-5$ & $3.036 \mathrm{E}+09$ & - & - & $3.0 \mathrm{E}+09$ & $1.222 \mathrm{E}-01$ & $1.150 \mathrm{E}-01$ & $1.230 \mathrm{E}-01$ & 0.085129 \\
\hline $11-1$ & $8.011 \mathrm{E}+07$ & - & - & - & $4.527 \mathrm{E}-04$ & $3.480 \mathrm{E}-04$ & - & 0.000205 \\
\hline $11-2$ & $8.414 \mathrm{E}+09$ & $7.802 \mathrm{E}+09$ & $7.350 \mathrm{E}+09$ & $8.2 \mathrm{E}+09$ & $6.370 \mathrm{E}-02$ & $5.780 \mathrm{E}-02$ & $6.260 \mathrm{E}-02$ & 0.033322 \\
\hline $11-4$ & $1.643 \mathrm{E}+10$ & - & - & $1.6 \mathrm{E}+10$ & $1.471 \mathrm{E}-01$ & $1.360 \mathrm{E}-01$ & $1.470 \mathrm{E}-01$ & 0.083713 \\
\hline $11-5$ & $1.363 \mathrm{E}+10$ & $1.445 \mathrm{E}+10$ & $1.104 \mathrm{E}+10$ & $1.4 \mathrm{E}+10$ & $1.243 \mathrm{E}-01$ & $1.060 \mathrm{E}-01$ & $1.270 \mathrm{E}-01$ & 0.071403 \\
\hline $12-1$ & $1.950 \mathrm{E}+08$ & - & - & - & $1.986 \mathrm{E}-03$ & $1.800 \mathrm{E}-03$ & - & 0.000852 \\
\hline $12-2$ & $1.017 \mathrm{E}+10$ & - & - & $1.0 \mathrm{E}+10$ & $1.365 \mathrm{E}-01$ & $1.210 \mathrm{E}-01$ & $1.380 \mathrm{E}-01$ & 0.067252 \\
\hline $12-3$ & $5.461 \mathrm{E}+10$ & $5.235 \mathrm{E}+10$ & $4.655 \mathrm{E}+10$ & $5.5 \mathrm{E}+10$ & $7.417 \mathrm{E}-01$ & $6.710 \mathrm{E}-01$ & $7.470 \mathrm{E}-01$ & 0.367456 \\
\hline $12-4$ & $3.285 \mathrm{E}+09$ & - & - & $3.3 \mathrm{E}+09$ & $5.164 \mathrm{E}-02$ & $5.010 \mathrm{E}-02$ & $5.190 \mathrm{E}-02$ & 0.027525 \\
\hline $12-5$ & $8.521 \mathrm{E}+09$ & - & - & $8.5 \mathrm{E}+09$ & $1.363 \mathrm{E}-01$ & $1.420 \mathrm{E}-01$ & $1.370 \mathrm{E}-01$ & 0.073279 \\
\hline $13-1$ & $4.119 \mathrm{E}+07$ & - & - & - & $2.011 \mathrm{E}-04$ & $1.960 \mathrm{E}-04$ & - & 0.000084 \\
\hline $13-2$ & $3.993 E+10$ & $3.980 \mathrm{E}+10$ & $3.470 \mathrm{E}+10$ & $4.0 \mathrm{E}+10$ & $2.554 \mathrm{E}-01$ & $2.360 \mathrm{E}-01$ & $2.590 \mathrm{E}-01$ & 0.122817 \\
\hline $13-4$ & $6.394 \mathrm{E}+09$ & - & - & $6.5 \mathrm{E}+09$ & $4.770 \mathrm{E}-02$ & $4.460 \mathrm{E}-02$ & $4.920 \mathrm{E}-02$ & 0.024770 \\
\hline $13-5$ & $2.934 \mathrm{E}+10$ & - & - & $2.9 \mathrm{E}+10$ & $2.226 \mathrm{E}-01$ & $2.020 \mathrm{E}-01$ & $2.220 \mathrm{E}-01$ & 0.116544 \\
\hline $14-6$ & $1.848 \mathrm{E}+08$ & - & - & - & $1.936 \mathrm{E}-03$ & - & - & 0.000842 \\
\hline $14-7$ & $5.559 \mathrm{E}+07$ & - & - & - & $6.017 \mathrm{E}-04$ & - & - & 0.000266 \\
\hline $14-8$ & $1.705 \mathrm{E}+07$ & - & - & - & $1.877 \mathrm{E}-04$ & - & - & 0.000084 \\
\hline $14-9$ & $4.711 \mathrm{E}+09$ & - & - & $4.7 \mathrm{E}+09$ & $8.495 \mathrm{E}-02$ & - & $8.490 \mathrm{E}-02$ & 0.048497 \\
\hline $14-10$ & $2.718 \mathrm{E}+10$ & $2.560 \mathrm{E}+10$ & $2.118 \mathrm{E}+10$ & $2.7 \mathrm{E}+10$ & $4.920 \mathrm{E}-01$ & - & $4.960 \mathrm{E}-01$ & 0.281384 \\
\hline $14-11$ & $3.253 \mathrm{E}+09$ & - & - & $3.2 \mathrm{E}+09$ & $8.648 \mathrm{E}-02$ & - & $8.570 \mathrm{E}-02$ & 0.059942 \\
\hline $14-12$ & $1.902 \mathrm{E}+10$ & - & - & $1.9 \mathrm{E}+10$ & $6.001 \mathrm{E}-01$ & - & $6.040 \mathrm{E}-01$ & 0.453124 \\
\hline $14-13$ & $2.540 \mathrm{E}+09$ & - & - & $2.6 \mathrm{E}+09$ & $8.649 \mathrm{E}-02$ & - & $8.820 \mathrm{E}-02$ & 0.067858 \\
\hline $15-7$ & $9.441 \mathrm{E}+06$ & - & - & - & $4.832 \mathrm{E}-05$ & - & - & 0.000021 \\
\hline $15-8$ & $6.022 \mathrm{E}+07$ & - & - & - & $3.133 \mathrm{E}-04$ & - & - & 0.000136 \\
\hline $15-9$ & $3.031 \mathrm{E}+10$ & - & - & $3.0 \mathrm{E}+10$ & $2.544 \mathrm{E}-01$ & - & $2.570 \mathrm{E}-01$ & 0.140089 \\
\hline $15-11$ & $1.132 \mathrm{E}+08$ & - & - & - & $1.380 \mathrm{E}-03$ & - & - & 0.000916 \\
\hline $15-12$ & $1.065 \mathrm{E}+10$ & - & - & $1.1 \mathrm{E}+10$ & $1.528 \mathrm{E}-01$ & - & $1.530 \mathrm{E}-01$ & 0.110074 \\
\hline $15-13$ & $1.766 \mathrm{E}+10$ & - & - & $1.8 \mathrm{E}+10$ & $2.727 \mathrm{E}-01$ & - & $2.740 \mathrm{E}-01$ & 0.203682 \\
\hline
\end{tabular}




\begin{tabular}{|c|c|c|c|c|c|c|}
\hline Levels & \multicolumn{2}{|c|}{$A_{i j}\left(\mathrm{~s}^{-1}\right)$} & \multicolumn{2}{|c|}{$S$} & \multicolumn{2}{|c|}{$g f$} \\
\hline$i-j$ & Present & MCDHF & Present & MCDHF & Present & MCDHF \\
\hline $3-1$ & $2.851 \mathrm{E}+06$ & $2.922 \mathrm{E}+06$ & 0.000253 & 0.000239 & $1.714 \mathrm{E}-04$ & $1.665 \mathrm{E}-04$ \\
\hline $3-2$ & $1.679 \mathrm{E}+06$ & $1.597 \mathrm{E}+06$ & 0.000206 & 0.000179 & $1.252 \mathrm{E}-04$ & $1.121 \mathrm{E}-04$ \\
\hline $4-2$ & $4.756 \mathrm{E}+05$ & $5.276 \mathrm{E}+05$ & 0.000102 & 0.000104 & $6.511 \mathrm{E}-05$ & $6.827 \mathrm{E}-05$ \\
\hline $5-2$ & $2.025 \mathrm{E}+06$ & $2.385 \mathrm{E}+06$ & 0.000549 & 0.000606 & $3.700 \mathrm{E}-04$ & $4.171 \mathrm{E}-04$ \\
\hline $6-1$ & $3.567 \mathrm{E}+09$ & $3.384 \mathrm{E}+09$ & 0.098161 & 0.096710 & $1.239 \mathrm{E}-01$ & $1.205 \mathrm{E}-01$ \\
\hline $6-2$ & $3.031 \mathrm{E}+08$ & $2.745 \mathrm{E}+08$ & 0.009881 & 0.009304 & $1.179 \mathrm{E}-02$ & $1.095 \mathrm{E}-02$ \\
\hline $7-2$ & $3.249 \mathrm{E}+09$ & $3.050 \mathrm{E}+09$ & 0.157308 & 0.153900 & $1.883 \mathrm{E}-01$ & $1.817 \mathrm{E}-01$ \\
\hline $8-1$ & $1.275 \mathrm{E}+10$ & $1.337 \mathrm{E}+10$ & 0.089567 & 0.096770 & $1.414 \mathrm{E}-01$ & $1.513 \mathrm{E}-01$ \\
\hline $8-2$ & $3.724 \mathrm{E}+09$ & $2.037 \mathrm{E}+09$ & 0.029939 & 0.016880 & $4.520 \mathrm{E}-02$ & $2.523 \mathrm{E}-02$ \\
\hline $9-1$ & $1.012 \mathrm{E}+10$ & $7.480 \mathrm{E}+09$ & 0.057772 & 0.045500 & $9.777 \mathrm{E}-02$ & $7.539 \mathrm{E}-02$ \\
\hline $9-2$ & $1.535 \mathrm{E}+10$ & $1.510 \mathrm{E}+10$ & 0.099343 & 0.104300 & $1.612 \mathrm{E}-01$ & $1.657 \mathrm{E}-01$ \\
\hline $10-1$ & $4.285 \mathrm{E}+09$ & $3.903 E+09$ & 0.045993 & 0.045080 & $7.945 \mathrm{E}-02$ & $7.599 \mathrm{E}-02$ \\
\hline $10-2$ & $2.322 \mathrm{E}+10$ & $2.131 E+10$ & 0.281844 & 0.279000 & $4.674 \mathrm{E}-01$ & $4.510 \mathrm{E}-01$ \\
\hline $11-3$ & $4.481 \mathrm{E}+09$ & $4.177 \mathrm{E}+09$ & 0.073667 & 0.070660 & $1.104 \mathrm{E}-01$ & $1.049 \mathrm{E}-01$ \\
\hline $11-4$ & $8.460 \mathrm{E}+09$ & $7.860 \mathrm{E}+09$ & 0.146762 & 0.140200 & $2.160 \mathrm{E}-01$ & $2.045 \mathrm{E}-01$ \\
\hline $11-5$ & $1.175 \mathrm{E}+10$ & $1.095 \mathrm{E}+10$ & 0.220574 & 0.210000 & $3.163 \mathrm{E}-01$ & $2.989 \mathrm{E}-01$ \\
\hline $11-6$ & $4.564 \mathrm{E}+05$ & $6.928 \mathrm{E}+05$ & 0.000033 & 0.000047 & $3.017 \mathrm{E}-05$ & $4.387 \mathrm{E}-05$ \\
\hline $11-7$ & $2.130 \mathrm{E}+05$ & $1.574 \mathrm{E}+05$ & 0.000016 & 0.000011 & $1.420 \mathrm{E}-05$ & $1.003 \mathrm{E}-05$ \\
\hline $11-8$ & $3.794 \mathrm{E}+05$ & $6.541 \mathrm{E}+05$ & 0.000098 & 0.000154 & $5.871 \mathrm{E}-05$ & $9.483 \mathrm{E}-05$ \\
\hline $11-9$ & $6.541 \mathrm{E}+05$ & $7.258 \mathrm{E}+05$ & 0.000318 & 0.000279 & $1.540 \mathrm{E}-04$ & $1.461 \mathrm{E}-04$ \\
\hline $11-10$ & $1.789 \mathrm{E}+06$ & $2.130 \mathrm{E}+06$ & 0.001089 & 0.000969 & $4.897 \mathrm{E}-04$ & $4.801 \mathrm{E}-04$ \\
\hline $12-3$ & $5.856 \mathrm{E}+05$ & $3.406 \mathrm{E}+05$ & 0.000005 & 0.000003 & $9.796 \mathrm{E}-06$ & $6.034 \mathrm{E}-06$ \\
\hline $12-4$ & $4.506 \mathrm{E}+07$ & $4.759 E+07$ & 0.000433 & 0.000499 & $7.763 \mathrm{E}-04$ & $8.687 \mathrm{E}-04$ \\
\hline $12-5$ & $5.927 \mathrm{E}+05$ & $2.073 \mathrm{E}+05$ & 0.000006 & 0.000002 & $1.066 \mathrm{E}-05$ & $3.941 \mathrm{E}-06$ \\
\hline $12-6$ & $5.679 \mathrm{E}+09$ & $5.087 \mathrm{E}+09$ & 0.166832 & 0.156000 & $2.060 \mathrm{E}-01$ & $1.899 \mathrm{E}-01$ \\
\hline $12-7$ & $1.568 \mathrm{E}+09$ & $1.379 \mathrm{E}+09$ & 0.046498 & 0.042590 & $5.724 \mathrm{E}-02$ & $5.173 \mathrm{E}-02$ \\
\hline $12-8$ & $5.984 \mathrm{E}+08$ & $7.277 \mathrm{E}+08$ & 0.042790 & 0.055140 & $3.929 \mathrm{E}-02$ & $4.966 \mathrm{E}-02$ \\
\hline $12-9$ & $1.633 \mathrm{E}+09$ & $1.333 \mathrm{E}+09$ & 0.173263 & 0.140300 & $1.395 \mathrm{E}-01$ & $1.132 \mathrm{E}-01$ \\
\hline $12-10$ & $1.333 \mathrm{E}+08$ & $1.328 \mathrm{E}+08$ & 0.016179 & 0.015600 & $1.245 \mathrm{E}-02$ & $1.214 \mathrm{E}-02$ \\
\hline $13-4$ & $2.593 \mathrm{E}+06$ & $2.465 \mathrm{E}+06$ & 0.000037 & 0.000038 & $6.637 \mathrm{E}-05$ & $6.703 \mathrm{E}-05$ \\
\hline $13-5$ & $8.441 \mathrm{E}+07$ & $8.541 \mathrm{E}+07$ & 0.001279 & 0.001413 & $2.255 \mathrm{E}-03$ & $2.419 \mathrm{E}-03$ \\
\hline $13-6$ & $8.076 \mathrm{E}+08$ & $7.357 \mathrm{E}+08$ & 0.034844 & 0.033360 & $4.334 \mathrm{E}-02$ & $4.080 \mathrm{E}-02$ \\
\hline $13-7$ & $7.062 \mathrm{E}+09$ & $6.296 \mathrm{E}+09$ & 0.307615 & 0.287500 & $3.814 \mathrm{E}-01$ & $3.509 \mathrm{E}-01$ \\
\hline $13-10$ & $1.808 \mathrm{E}+09$ & $1.667 \mathrm{E}+09$ & 0.318270 & 0.287300 & $2.477 \mathrm{E}-01$ & $2.252 \mathrm{E}-01$ \\
\hline $14-3$ & $1.744 \mathrm{E}+07$ & $1.823 \mathrm{E}+07$ & 0.000051 & 0.000057 & $1.072 \mathrm{E}-04$ & $1.184 \mathrm{E}-04$ \\
\hline $14-4$ & $3.495 \mathrm{E}+06$ & $3.255 \mathrm{E}+06$ & 0.000011 & 0.000011 & $2.203 \mathrm{E}-05$ & $2.168 \mathrm{E}-05$ \\
\hline $14-6$ & $1.332 \mathrm{E}+10$ & $1.180 \mathrm{E}+10$ & 0.101300 & 0.093950 & $1.558 \mathrm{E}-01$ & $1.423 \mathrm{E}-01$ \\
\hline
\end{tabular}


Table 5: Present collision strengths $\Omega$ of some transitions for Ar XII (compared to E05: The results of Eissner et al. [21]) and for Ar XIV (compared to B86: The results of Bhatia et al. [19])

\begin{tabular}{|c|c|c|c|c|c|c|c|c|}
\hline \multirow{3}{*}{$\begin{array}{l}\text { Trans. } \\
i-j\end{array}$} & \multicolumn{6}{|c|}{ Ar XII } & \multirow{2}{*}{\multicolumn{2}{|c|}{$\begin{array}{l}\text { Ar XIV } \\
40 \text { Ryd }\end{array}$}} \\
\hline & \multicolumn{2}{|c|}{35 Ryd } & \multicolumn{2}{|c|}{70 Ryd } & \multicolumn{2}{|c|}{105 Ryd } & & \\
\hline & Present & E05 & Present & E05 & Present & E05 & Present & B86 \\
\hline $1-2$ & $3.486 \mathrm{E}-02$ & $3.358 \mathrm{E}-02$ & $1.686 \mathrm{E}-02$ & $1.675 \mathrm{E}-02$ & $1.005 \mathrm{E}-02$ & $1.007 \mathrm{E}-02$ & $5.058 \mathrm{E}-02$ & $4.733 \mathrm{E}-02$ \\
\hline $1-3$ & $5.122 \mathrm{E}-02$ & $4.925 \mathrm{E}-02$ & $2.526 \mathrm{E}-02$ & $2.503 \mathrm{E}-02$ & $1.537 \mathrm{E}-02$ & $1.534 \mathrm{E}-02$ & $6.127 \mathrm{E}-03$ & $6.382 \mathrm{E}-03$ \\
\hline $1-4$ & $9.773 \mathrm{E}-03$ & $9.588 \mathrm{E}-03$ & $4.024 \mathrm{E}-03$ & $4.063 \mathrm{E}-03$ & $2.091 \mathrm{E}-03$ & $2.126 \mathrm{E}-03$ & $6.664 \mathrm{E}-03$ & $6.484 \mathrm{E}-03$ \\
\hline $1-5$ & $1.867 \mathrm{E}-02$ & $1.834 \mathrm{E}-02$ & $7.808 \mathrm{E}-03$ & $7.909 E-03$ & $4.111 \mathrm{E}-03$ & $4.206 \mathrm{E}-03$ & $4.334 \mathrm{E}-03$ & $4.207 \mathrm{E}-03$ \\
\hline $1-6$ & $1.217 \mathrm{E}+00$ & $1.265 \mathrm{E}+00$ & $1.237 \mathrm{E}+00$ & $1.479 \mathrm{E}+00$ & $1.194 \mathrm{E}+00$ & $1.618 \mathrm{E}+00$ & $5.498 \mathrm{E}-01$ & $5.380 \mathrm{E}-01$ \\
\hline $1-7$ & $8.144 \mathrm{E}-01$ & $8.407 \mathrm{E}-01$ & $8.331 \mathrm{E}-01$ & $9.792 \mathrm{E}-01$ & $8.058 \mathrm{E}-01$ & $1.070 \mathrm{E}+00$ & $6.857 \mathrm{E}-03$ & $6.185 \mathrm{E}-03$ \\
\hline $1-8$ & $4.101 \mathrm{E}-01$ & $4.223 \mathrm{E}-01$ & $4.201 \mathrm{E}-01$ & $4.910 \mathrm{E}-01$ & $4.064 \mathrm{E}-01$ & $5.364 \mathrm{E}-01$ & $4.801 \mathrm{E}-01$ & $4.401 \mathrm{E}-01$ \\
\hline $1-9$ & $4.860 \mathrm{E}-04$ & $5.406 \mathrm{E}-04$ & $3.970 \mathrm{E}-04$ & $4.403 \mathrm{E}-04$ & $3.550 \mathrm{E}-04$ & $4.214 \mathrm{E}-04$ & $2.993 \mathrm{E}-01$ & $2.973 \mathrm{E}-01$ \\
\hline $1-10$ & $1.420 \mathrm{E}-04$ & $1.804 \mathrm{E}-04$ & $9.600 \mathrm{E}-05$ & $8.258 \mathrm{E}-05$ & $8.100 \mathrm{E}-05$ & $6.156 \mathrm{E}-05$ & $2.398 \mathrm{E}-01$ & $2.263 \mathrm{E}-01$ \\
\hline $2-3$ & $7.236 \mathrm{E}-02$ & $8.525 \mathrm{E}-02$ & $4.175 \mathrm{E}-02$ & $5.886 \mathrm{E}-02$ & $3.008 \mathrm{E}-02$ & $4.862 \mathrm{E}-02$ & $3.677 \mathrm{E}-03$ & $4.028 \mathrm{E}-03$ \\
\hline $2-4$ & $5.294 \mathrm{E}-02$ & $5.168 \mathrm{E}-02$ & $5.654 \mathrm{E}-02$ & $5.559 \mathrm{E}-02$ & $5.913 \mathrm{E}-02$ & $5.851 \mathrm{E}-02$ & $8.699 \mathrm{E}-03$ & $8.598 \mathrm{E}-03$ \\
\hline $2-5$ & $5.022 \mathrm{E}-02$ & $4.991 \mathrm{E}-02$ & $4.632 \mathrm{E}-02$ & $4.631 \mathrm{E}-02$ & $4.596 \mathrm{E}-02$ & 4.607E-02 & $2.095 \mathrm{E}-02$ & $2.095 \mathrm{E}-02$ \\
\hline $2-6$ & $9.837 \mathrm{E}-03$ & $9.980 \mathrm{E}-03$ & $6.645 \mathrm{E}-03$ & $7.501 \mathrm{E}-03$ & $5.197 \mathrm{E}-03$ & $6.544 \mathrm{E}-03$ & $6.574 \mathrm{E}-02$ & $6.485 \mathrm{E}-02$ \\
\hline $2-7$ & $7.486 \mathrm{E}-03$ & $7.457 \mathrm{E}-03$ & $3.846 \mathrm{E}-03$ & $3.926 \mathrm{E}-03$ & $2.350 \mathrm{E}-03$ & $2.410 \mathrm{E}-03$ & $8.949 \mathrm{E}-01$ & $8.954 \mathrm{E}-01$ \\
\hline $2-8$ & $4.719 \mathrm{E}-03$ & $4.724 \mathrm{E}-03$ & $2.619 \mathrm{E}-03$ & $2.770 \mathrm{E}-03$ & $1.742 \mathrm{E}-03$ & $1.947 \mathrm{E}-03$ & $1.654 \mathrm{E}-01$ & $1.641 \mathrm{E}-01$ \\
\hline $2-9$ & $1.173 \mathrm{E}+00$ & $1.189 \mathrm{E}+00$ & $1.243 \mathrm{E}+00$ & $1.403 \mathrm{E}+00$ & $1.218 \mathrm{E}+00$ & $1.542 \mathrm{E}+00$ & $5.302 \mathrm{E}-01$ & $4.894 \mathrm{E}-01$ \\
\hline $2-10$ & $3.821 \mathrm{E}-02$ & $3.848 \mathrm{E}-02$ & $3.282 \mathrm{E}-02$ & $3.641 \mathrm{E}-02$ & $2.944 \mathrm{E}-02$ & $3.650 \mathrm{E}-02$ & $1.481 \mathrm{E}+00$ & $1.399 \mathrm{E}+00$ \\
\hline $3-4$ & $4.458 \mathrm{E}-02$ & $4.349 \mathrm{E}-02$ & $4.318 \mathrm{E}-02$ & $4.252 \mathrm{E}-02$ & $4.375 \mathrm{E}-02$ & $4.334 \mathrm{E}-02$ & $3.288 \mathrm{E}-02$ & $3.189 \mathrm{E}-02$ \\
\hline $3-5$ & $1.280 \mathrm{E}-01$ & $1.273 \mathrm{E}-01$ & $1.320 \mathrm{E}-01$ & $1.328 \mathrm{E}-01$ & $1.365 \mathrm{E}-01$ & $1.382 \mathrm{E}-01$ & $3.379 \mathrm{E}-02$ & $3.145 \mathrm{E}-02$ \\
\hline $3-6$ & $2.351 \mathrm{E}-02$ & $2.348 \mathrm{E}-02$ & $1.493 \mathrm{E}-02$ & $1.616 \mathrm{E}-02$ & $1.118 \mathrm{E}-02$ & $1.319 \mathrm{E}-02$ & $1.326 \mathrm{E}-02$ & $1.276 \mathrm{E}-02$ \\
\hline $3-7$ & $5.883 \mathrm{E}-03$ & $5.906 \mathrm{E}-03$ & $3.157 \mathrm{E}-03$ & $3.298 \mathrm{E}-03$ & $2.030 \mathrm{E}-03$ & $2.197 \mathrm{E}-03$ & $7.016 \mathrm{E}-03$ & $6.786 \mathrm{E}-03$ \\
\hline $3-8$ & $7.240 \mathrm{E}-04$ & $7.432 \mathrm{E}-04$ & $3.690 \mathrm{E}-04$ & $3.833 \mathrm{E}-04$ & $2.250 \mathrm{E}-04$ & $2.337 \mathrm{E}-04$ & $2.981 \mathrm{E}-03$ & $2.853 \mathrm{E}-03$ \\
\hline $3-9$ & $8.207 \mathrm{E}-02$ & $8.488 \mathrm{E}-02$ & $7.753 \mathrm{E}-02$ & $8.881 \mathrm{E}-02$ & $7.248 \mathrm{E}-02$ & $9.308 \mathrm{E}-02$ & $5.950 \mathrm{E}-04$ & $5.700 \mathrm{E}-04$ \\
\hline $3-10$ & $1.658 \mathrm{E}+00$ & $1.684 \mathrm{E}+00$ & $1.745 \mathrm{E}+00$ & $1.980 \mathrm{E}+00$ & $1.705 \mathrm{E}+00$ & $2.174 \mathrm{E}+00$ & $1.666 \mathrm{E}-03$ & $1.621 \mathrm{E}-03$ \\
\hline $4-5$ & $2.790 \mathrm{E}-02$ & $2.713 \mathrm{E}-02$ & $1.617 \mathrm{E}-02$ & $1.605 \mathrm{E}-02$ & $1.170 \mathrm{E}-02$ & $1.164 \mathrm{E}-02$ & $7.751 \mathrm{E}-02$ & $7.355 \mathrm{E}-02$ \\
\hline $4-6$ & $2.322 \mathrm{E}-03$ & $2.357 \mathrm{E}-03$ & $1.206 \mathrm{E}-03$ & $1.247 \mathrm{E}-03$ & $7.420 \mathrm{E}-04$ & $7.689 \mathrm{E}-04$ & $1.961 \mathrm{E}-02$ & $1.896 \mathrm{E}-02$ \\
\hline $4-7$ & $4.810 \mathrm{E}-03$ & $4.828 \mathrm{E}-03$ & $2.516 \mathrm{E}-03$ & $2.583 \mathrm{E}-03$ & $1.556 \mathrm{E}-03$ & $1.610 \mathrm{E}-03$ & $2.148 \mathrm{E}-02$ & $2.066 \mathrm{E}-02$ \\
\hline $4-8$ & $4.701 \mathrm{E}-03$ & $4.720 \mathrm{E}-03$ & $2.967 \mathrm{E}-03$ & $3.233 \mathrm{E}-03$ & $2.204 \mathrm{E}-03$ & $2.621 \mathrm{E}-03$ & $5.158 \mathrm{E}-03$ & $4.988 \mathrm{E}-03$ \\
\hline $4-9$ & $1.784 \mathrm{E}-01$ & $1.861 \mathrm{E}-01$ & $1.854 \mathrm{E}-01$ & $2.181 \mathrm{E}-01$ & $1.804 \mathrm{E}-01$ & $2.390 \mathrm{E}-01$ & $2.026 \mathrm{E}-03$ & $1.911 \mathrm{E}-03$ \\
\hline $4-10$ & $6.135 \mathrm{E}-03$ & $6.226 \mathrm{E}-03$ & $3.189 \mathrm{E}-03$ & $3.325 \mathrm{E}-03$ & $1.954 \mathrm{E}-03$ & $2.059 \mathrm{E}-03$ & $3.987 \mathrm{E}-03$ & $3.841 \mathrm{E}-03$ \\
\hline $5-6$ & $1.241 \mathrm{E}-02$ & $1.300 \mathrm{E}-02$ & $8.092 \mathrm{E}-03$ & $9.567 \mathrm{E}-03$ & $6.158 \mathrm{E}-03$ & $8.192 \mathrm{E}-03$ & $1.627 \mathrm{E}-02$ & $1.542 \mathrm{E}-02$ \\
\hline $5-7$ & $1.261 \mathrm{E}-02$ & $1.306 \mathrm{E}-02$ & $8.999 \mathrm{E}-03$ & $1.061 \mathrm{E}-02$ & $7.295 \mathrm{E}-03$ & $9.701 \mathrm{E}-03$ & $4.674 \mathrm{E}-02$ & $4.500 \mathrm{E}-02$ \\
\hline $5-8$ & $4.151 \mathrm{E}-03$ & $4.175 \mathrm{E}-03$ & $2.147 \mathrm{E}-03$ & $2.210 \mathrm{E}-03$ & $1.318 \mathrm{E}-03$ & $1.365 \mathrm{E}-03$ & $6.603 \mathrm{E}-03$ & $6.421 \mathrm{E}-03$ \\
\hline $5-9$ & $1.248 \mathrm{E}-02$ & $1.263 \mathrm{E}-02$ & $9.104 \mathrm{E}-03$ & $1.010 \mathrm{E}-02$ & $7.468 \mathrm{E}-03$ & $9.219 \mathrm{E}-03$ & $5.227 \mathrm{E}-03$ & $4.974 \mathrm{E}-03$ \\
\hline $5-10$ & $4.532 \mathrm{E}-01$ & $4.708 \mathrm{E}-01$ & $4.687 \mathrm{E}-01$ & $5.522 \mathrm{E}-01$ & $4.550 \mathrm{E}-01$ & $6.052 \mathrm{E}-01$ & $6.365 \mathrm{E}-03$ & $6.106 \mathrm{E}-03$ \\
\hline $6-7$ & $9.390 \mathrm{E}-02$ & $9.333 \mathrm{E}-02$ & $6.855 \mathrm{E}-02$ & $6.879 \mathrm{E}-02$ & $5.986 \mathrm{E}-02$ & $6.025 \mathrm{E}-02$ & $4.131 \mathrm{E}-02$ & $3.912 \mathrm{E}-02$ \\
\hline $6-8$ & $3.644 \mathrm{E}-02$ & $3.611 \mathrm{E}-02$ & $3.413 \mathrm{E}-02$ & $3.406 \mathrm{E}-02$ & $3.404 \mathrm{E}-02$ & $3.412 \mathrm{E}-02$ & $4.169 \mathrm{E}-02$ & $3.804 \mathrm{E}-02$ \\
\hline $6-9$ & $2.344 \mathrm{E}-02$ & $2.280 \mathrm{E}-02$ & $1.094 \mathrm{E}-02$ & $1.090 \mathrm{E}-02$ & $6.355 \mathrm{E}-03$ & $6.362 \mathrm{E}-03$ & $1.030 \mathrm{E}-02$ & $9.923 \mathrm{E}-03$ \\
\hline $6-10$ & $6.161 \mathrm{E}-02$ & $6.018 \mathrm{E}-02$ & $2.820 \mathrm{E}-02$ & $2.820 \mathrm{E}-02$ & $1.622 \mathrm{E}-02$ & $1.629 \mathrm{E}-02$ & $1.959 \mathrm{E}-02$ & $1.922 \mathrm{E}-02$ \\
\hline $7-8$ & $4.313 \mathrm{E}-02$ & $4.302 \mathrm{E}-02$ & $2.297 \mathrm{E}-02$ & $2.324 \mathrm{E}-02$ & $1.526 \mathrm{E}-02$ & $1.548 \mathrm{E}-02$ & $6.014 \mathrm{E}-02$ & $5.523 \mathrm{E}-02$ \\
\hline $7-9$ & $2.823 \mathrm{E}-02$ & $2.752 \mathrm{E}-02$ & $1.271 \mathrm{E}-02$ & $1.270 \mathrm{E}-02$ & $7.135 \mathrm{E}-03$ & $7.166 \mathrm{E}-03$ & $2.130 \mathrm{E}-02$ & $1.958 \mathrm{E}-02$ \\
\hline $7-10$ & $2.884 \mathrm{E}-02$ & $2.811 \mathrm{E}-02$ & $1.332 \mathrm{E}-02$ & $1.330 \mathrm{E}-02$ & $7.682 \mathrm{E}-03$ & $7.706 \mathrm{E}-03$ & $2.397 \mathrm{E}-02$ & $2.422 \mathrm{E}-02$ \\
\hline $8-9$ & $1.693 \mathrm{E}-02$ & $1.655 \mathrm{E}-02$ & $7.668 \mathrm{E}-03$ & $7.678 \mathrm{E}-03$ & $4.365 \mathrm{E}-03$ & $4.390 \mathrm{E}-03$ & $5.504 \mathrm{E}-03$ & $5.382 \mathrm{E}-03$ \\
\hline $8-10$ & $1.167 \mathrm{E}-02$ & $1.134 \mathrm{E}-02$ & $5.437 \mathrm{E}-03$ & $5.412 \mathrm{E}-03$ & $3.142 \mathrm{E}-03$ & $3.145 \mathrm{E}-03$ & $9.142 \mathrm{E}-03$ & $8.623 \mathrm{E}-03$ \\
\hline $9-10$ & $4.766 \mathrm{E}-02$ & $4.773 \mathrm{E}-02$ & $3.846 \mathrm{E}-02$ & $3.848 \mathrm{E}-02$ & $3.560 \mathrm{E}-02$ & $3.570 \mathrm{E}-02$ & $4.458 \mathrm{E}-02$ & $4.210 \mathrm{E}-02$ \\
\hline
\end{tabular}


Table 6: Stark line widths for 12 Ar XII lines at electron density $N=10^{18} \mathrm{~cm}^{-3}$ at different temperatures, the wavelengths $\lambda$ are taken from the SUPERSTRUCTURE (SST) code of Eissner et al. [34].

\begin{tabular}{|c|c|c|c|c|c|}
\hline Transition & $T\left(10^{5} \mathrm{~K}\right)$ & $W(\mathrm{pm})$ & Transition & $T\left(10^{5} \mathrm{~K}\right)$ & $W(\mathrm{pm})$ \\
\hline \multirow{6}{*}{$\begin{array}{c}2 \mathrm{~s}^{2} 2 \mathrm{p}^{3}{ }^{4} \mathrm{~S}_{3 / 2}^{\circ}-2 \mathrm{~s}^{2} 2 \mathrm{p}^{2}\left({ }^{3} \mathrm{P}\right) 3 \mathrm{~d}^{4} \mathrm{P}_{5 / 2} \\
\lambda=31.26 \AA \\
1-54\end{array}$} & 5 & $1.709 \mathrm{E}-03$ & \multirow{6}{*}{$\begin{array}{c}2 \mathrm{~s}^{2} 2 \mathrm{p}^{3}{ }^{2} \mathrm{P}_{3 / 2}^{\circ}-2 \mathrm{~s}^{2} 2 \mathrm{p}^{2}\left({ }^{1} \mathrm{D}\right) 3 \mathrm{~s}^{2} \mathrm{D}_{5 / 2} \\
\lambda=35.53 \AA \\
5-21\end{array}$} & 5 & $1.045 \mathrm{E}-03$ \\
\hline & 10 & $1.194 \mathrm{E}-03$ & & 10 & $7.048 \mathrm{E}-04$ \\
\hline & 15 & $9.543 \mathrm{E}-04$ & & 15 & $5.592 \mathrm{E}-04$ \\
\hline & 20 & $8.026 \mathrm{E}-04$ & & 20 & $4.746 \mathrm{E}-04$ \\
\hline & 25 & $6.946 \mathrm{E}-04$ & & 25 & $4.179 \mathrm{E}-04$ \\
\hline & 50 & $4.211 \mathrm{E}-04$ & & 50 & $2.805 \mathrm{E}-04$ \\
\hline \multirow{6}{*}{$\begin{array}{c}2 \mathrm{~s}^{2} 2 \mathrm{p}^{3}{ }^{4} \mathrm{~S}_{3 / 2}^{\circ}-2 \mathrm{~s}^{2} 2 \mathrm{p}^{2}\left({ }^{3} \mathrm{P}\right) 3 \mathrm{~s}^{4} \mathrm{P}_{5 / 2} \\
\lambda=34.52 \AA \\
1-18\end{array}$} & 5 & $1.020 \mathrm{E}-03$ & \multirow{6}{*}{$\begin{array}{c}2 \mathrm{~s}^{2} 2 \mathrm{p}^{3}{ }^{2} \mathrm{P}_{3 / 2}^{\circ}-2 \mathrm{~s}^{2} 2 \mathrm{p}^{2}\left({ }^{3} \mathrm{P}\right) 3 \mathrm{~s}^{2} \mathrm{P}_{3 / 2} \\
\lambda=36.13 \AA \\
5-20\end{array}$} & 5 & $1.047 \mathrm{E}-03$ \\
\hline & 10 & $6.821 \mathrm{E}-04$ & & 10 & $7.157 \mathrm{E}-04$ \\
\hline & 15 & $5.419 \mathrm{E}-04$ & & 15 & $5.718 \mathrm{E}-04$ \\
\hline & 20 & 4.604E-04 & & 20 & $4.873 \mathrm{E}-04$ \\
\hline & 25 & $4.054 \mathrm{E}-04$ & & 25 & $4.301 \mathrm{E}-04$ \\
\hline & 50 & $2.712 \mathrm{E}-04$ & & 50 & $2.899 \mathrm{E}-04$ \\
\hline \multirow{6}{*}{$\begin{array}{c}2 \mathrm{~s}^{2} 2 \mathrm{p}^{3}{ }^{4} \mathrm{~S}_{3 / 2}^{\circ}-2 \mathrm{~s}^{2} 2 \mathrm{p}^{2}\left({ }^{3} \mathrm{P}\right) 3 \mathrm{~s}^{4} \mathrm{P}_{3 / 2} \\
\lambda=34.65 \AA \\
1-17\end{array}$} & 5 & $1.388 \mathrm{E}-03$ & \multirow{6}{*}{$\begin{array}{c}2 \mathrm{~s}^{2} 2 \mathrm{p}^{3}{ }^{2} \mathrm{P}^{\circ}{ }_{1 / 2}-2 s\left({ }^{2} \mathrm{~S}\right) 2 \mathrm{p}^{4}\left({ }^{1} \mathrm{~S}\right){ }^{2} \mathrm{~S}_{1 / 2} \\
\lambda=172.82 \AA \\
4-11\end{array}$} & 5 & $3.939 \mathrm{E}-03$ \\
\hline & 10 & $8.349 \mathrm{E}-04$ & & 10 & $3.080 \mathrm{E}-03$ \\
\hline & 15 & $6.318 \mathrm{E}-04$ & & 15 & $2.888 \mathrm{E}-03$ \\
\hline & 20 & $5.219 \mathrm{E}-04$ & & 20 & $2.822 \mathrm{E}-03$ \\
\hline & 25 & $4.512 \mathrm{E}-04$ & & 25 & $2.766 \mathrm{E}-03$ \\
\hline & 50 & $2.898 \mathrm{E}-04$ & & 50 & $2.383 \mathrm{E}-03$ \\
\hline \multirow{6}{*}{$\begin{array}{c}2 \mathrm{~s}^{2} 2 \mathrm{p}^{3}{ }^{4} \mathrm{~S}^{\circ}{ }_{3 / 2}-2 \mathrm{~s}^{2} 2 \mathrm{p}^{2}\left({ }^{3} \mathrm{P}\right) 3 \mathrm{~s}^{4} \mathrm{P}_{1 / 2} \\
\lambda=34.75 \AA \\
1-16\end{array}$} & 5 & $1.779 \mathrm{E}-03$ & \multirow{6}{*}{$\begin{array}{c}2 \mathrm{~s}^{2} 2 \mathrm{p}^{3}{ }^{4} \mathrm{~S}^{\circ}{ }_{3 / 2}-2 \mathrm{~s}\left({ }^{2} \mathrm{~S}\right) 2 \mathrm{p}^{4}\left({ }^{3} \mathrm{P}\right){ }^{4} \mathrm{P}_{1 / 2} \\
\lambda=214.91 \AA \\
1-8\end{array}$} & 5 & $5.329 \mathrm{E}-03$ \\
\hline & 10 & $9.961 \mathrm{E}-04$ & & 10 & $3.840 \mathrm{E}-03$ \\
\hline & 15 & $7.260 \mathrm{E}-04$ & & 15 & $3.215 \mathrm{E}-03$ \\
\hline & 20 & $5.859 \mathrm{E}-04$ & & 20 & $2.859 \mathrm{E}-03$ \\
\hline & 25 & $4.987 \mathrm{E}-04$ & & 25 & $2.621 \mathrm{E}-03$ \\
\hline & 50 & $3.086 \mathrm{E}-04$ & & 50 & $2.020 \mathrm{E}-03$ \\
\hline \multirow{6}{*}{$\begin{array}{c}2 \mathrm{~s}^{2} 2 \mathrm{p}^{32} \mathrm{D}_{3 / 2}^{\circ}-2 \mathrm{~s}^{2} 2 \mathrm{p}^{2}\left({ }^{1} \mathrm{D}\right) 3 \mathrm{~s}^{2} \mathrm{D}_{3 / 2} \\
\lambda=34.82 \AA \\
2-22\end{array}$} & 5 & $1.078 \mathrm{E}-03$ & \multirow{6}{*}{$\begin{array}{c}2 \mathrm{~s}^{2} 2 \mathrm{p}^{3}{ }^{4} \mathrm{~S}_{3 / 2}^{\circ}-2 \mathrm{~s}\left({ }^{2} \mathrm{~S}\right) 2 \mathrm{p}^{4}\left({ }^{3} \mathrm{P}\right){ }^{4} \mathrm{P}_{3 / 2} \\
\lambda=217.87 \AA \\
1-7\end{array}$} & 5 & $5.453 \mathrm{E}-03$ \\
\hline & 10 & $7.079 \mathrm{E}-04$ & & 10 & $3.932 \mathrm{E}-03$ \\
\hline & 15 & $5.583 \mathrm{E}-04$ & & 15 & $3.295 \mathrm{E}-03$ \\
\hline & 20 & $4.728 \mathrm{E}-04$ & & 20 & $2.933 \mathrm{E}-03$ \\
\hline & 25 & $4.158 \mathrm{E}-04$ & & 25 & $2.690 \mathrm{E}-03$ \\
\hline & 50 & $2.785 \mathrm{E}-04$ & & 50 & $2.074 \mathrm{E}-03$ \\
\hline \multirow{6}{*}{$\begin{array}{c}2 \mathrm{~s}^{2} 2 \mathrm{p}^{32} \mathrm{P}_{3 / 2}^{\circ}-2 \mathrm{~s}^{2} 2 \mathrm{p}^{2}\left({ }^{1} \mathrm{D}\right) 3 \mathrm{~s}^{2} \mathrm{D}_{3 / 2} \\
\lambda=35.52 \AA \\
5-22\end{array}$} & 5 & $1.136 \mathrm{E}-03$ & \multirow{6}{*}{$\begin{array}{c}2 \mathrm{~s}^{2} 2 \mathrm{p}^{3}{ }^{4} \mathrm{~S}_{3 / 2}^{\circ}-2 \mathrm{~s}\left({ }^{2} \mathrm{~S}\right) 2 \mathrm{p}^{4}\left({ }^{3} \mathrm{P}\right){ }^{4} \mathrm{P}_{5 / 2} \\
\lambda=223.88 \AA \\
1-6\end{array}$} & 5 & $5.656 \mathrm{E}-03$ \\
\hline & 10 & $7.425 \mathrm{E}-04$ & & 10 & $4.083 \mathrm{E}-03$ \\
\hline & 15 & $5.814 \mathrm{E}-04$ & & 15 & $3.425 \mathrm{E}-03$ \\
\hline & 20 & $4.899 \mathrm{E}-04$ & & 20 & $3.051 \mathrm{E}-03$ \\
\hline & 25 & $4.293 \mathrm{E}-04$ & & 25 & $2.801 \mathrm{E}-03$ \\
\hline & 50 & $2.853 \mathrm{E}-04$ & & 50 & $2.166 \mathrm{E}-03$ \\
\hline
\end{tabular}


Table 7: Same as in Table 6 but for the Ar XIV ion.

\begin{tabular}{|c|c|c|c|c|c|}
\hline Transition & $T\left(10^{5} \mathrm{~K}\right)$ & $W(\mathrm{pm})$ & Transition & $T\left(10^{5} \mathrm{~K}\right)$ & $W(\mathrm{pm})$ \\
\hline \multirow{6}{*}{$\begin{array}{c}2 \mathrm{~s}^{2} 2 \mathrm{p}^{2} \mathrm{P}^{\circ}{ }_{1 / 2}-2 \mathrm{~s}^{2} 3 \mathrm{~d}^{2} \mathrm{D}_{3 / 2} \\
\lambda=27.15 \AA \\
1-22\end{array}$} & 5 & $2.044 \mathrm{E}-04$ & \multirow{6}{*}{$\begin{array}{c}2 \mathrm{~s}^{2} 2 \mathrm{p}^{2} \mathrm{P}_{1 / 2}^{\circ}-2 \mathrm{~s} 2 \mathrm{p}^{2}{ }^{2} \mathrm{~S}_{1 / 2} \\
\lambda=192.36 \AA \\
1-8\end{array}$} & 5 & $5.923 \mathrm{E}-03$ \\
\hline & 10 & $1.442 \mathrm{E}-04$ & & 10 & $4.369 \mathrm{E}-03$ \\
\hline & 15 & $1.174 \mathrm{E}-04$ & & 15 & $3.685 \mathrm{E}-03$ \\
\hline & 20 & $1.013 \mathrm{E}-04$ & & 20 & $3.284 \mathrm{E}-03$ \\
\hline & 25 & $9.027 \mathrm{E}-05$ & & 25 & $3.011 \mathrm{E}-03$ \\
\hline & 50 & $6.273 \mathrm{E}-05$ & & 50 & $2.298 \mathrm{E}-03$ \\
\hline \multirow{6}{*}{$\begin{array}{c}2 \mathrm{~s}^{2} 2 \mathrm{p}^{2} \mathrm{P}^{\circ} 3 / 2-2 \mathrm{~s}^{2} 3 \mathrm{~d}^{2} \mathrm{D}_{5 / 2} \\
\lambda=27.31 \AA \\
2-23\end{array}$} & 5 & $6.377 \mathrm{E}-04$ & \multirow{6}{*}{$\begin{array}{c}2 \mathrm{~s} 2 \mathrm{p}^{2}{ }^{2} \mathrm{D}_{3 / 2}-2 \mathrm{p}^{3}{ }^{2} \mathrm{P}^{\circ}{ }_{1 / 2} \\
\lambda=197.51 \AA \\
6-14\end{array}$} & 5 & $6.969 \mathrm{E}-03$ \\
\hline & 10 & $4.527 \mathrm{E}-04$ & & 10 & $5.038 \mathrm{E}-03$ \\
\hline & 15 & $2.036 \mathrm{E}-04$ & & 15 & $4.181 \mathrm{E}-03$ \\
\hline & 20 & $1.437 \mathrm{E}-04$ & & 20 & $3.665 \mathrm{E}-03$ \\
\hline & 25 & $1.170 \mathrm{E}-04$ & & 25 & $3.307 \mathrm{E}-03$ \\
\hline & 50 & $1.010 \mathrm{E}-04$ & & 50 & $2.378 \mathrm{E}-03$ \\
\hline \multirow{6}{*}{$\begin{array}{c}2 \mathrm{~s} 2 \mathrm{p}^{2}{ }^{2} \mathrm{D}_{5 / 2}-2 \mathrm{~s} 2 \mathrm{p}\left({ }^{3} \mathrm{P}^{\circ}\right) 3 \mathrm{~s}^{2} \mathrm{P}^{\circ} 3 / 2 \\
\lambda=30.17 \AA \\
7-25\end{array}$} & 5 & $1.618 \mathrm{E}-03$ & \multirow{6}{*}{$\begin{array}{c}2 \mathrm{~s} 2 \mathrm{p}^{2}{ }^{4} \mathrm{P}_{5 / 2}-2 \mathrm{p}^{3}{ }^{4} \mathrm{~S}^{\circ} 3 / 2 \\
\lambda=211.85 \AA \\
5-11\end{array}$} & 5 & $4.873 \mathrm{E}-03$ \\
\hline & 10 & $1.144 \mathrm{E}-03$ & & 10 & $3.490 \mathrm{E}-03$ \\
\hline & 15 & $5.088 \mathrm{E}-04$ & & 15 & $2.878 \mathrm{E}-03$ \\
\hline & 20 & $3.552 \mathrm{E}-04$ & & 20 & $2.512 \mathrm{E}-03$ \\
\hline & 25 & $2.865 \mathrm{E}-04$ & & 25 & $2.260 \mathrm{E}-03$ \\
\hline & 50 & $2.452 \mathrm{E}-04$ & & 50 & $1.613 \mathrm{E}-03$ \\
\hline \multirow{6}{*}{$\begin{array}{c}2 \mathrm{~s}^{2} 2 \mathrm{p}^{2} \mathrm{P}_{1 / 2}^{\circ}-2 \mathrm{~s} 2 \mathrm{p}^{2}{ }^{2} \mathrm{P}_{1 / 2} \\
\lambda=179.49 \AA \\
1-9\end{array}$} & 5 & $8.238 \mathrm{E}-03$ & \multirow{6}{*}{$\begin{array}{c}2 \mathrm{~s}^{2} 2 \mathrm{p}^{2} \mathrm{P}^{\circ}{ }_{1 / 2}-2 \mathrm{~s} 2 \mathrm{p}^{2}{ }^{2} \mathrm{D}_{3 / 2} \\
\lambda=240.66 \AA \\
1-6\end{array}$} & 5 & $8.087 \mathrm{E}-03$ \\
\hline & 10 & $5.983 \mathrm{E}-03$ & & 10 & $6.300 \mathrm{E}-03$ \\
\hline & 15 & $4.981 \mathrm{E}-03$ & & 15 & $5.407 \mathrm{E}-03$ \\
\hline & 20 & $4.377 \mathrm{E}-03$ & & 20 & $4.850 \mathrm{E}-03$ \\
\hline & 25 & $3.960 \mathrm{E}-03$ & & 25 & $4.457 \mathrm{E}-03$ \\
\hline & 50 & $2.879 \mathrm{E}-03$ & & 50 & $3.398 \mathrm{E}-03$ \\
\hline \multirow{6}{*}{$\begin{array}{c}2 \mathrm{~s}^{2} 2 \mathrm{p}^{2} \mathrm{P}_{3 / 2}^{\circ}-2 \mathrm{~s} 2 \mathrm{p}^{2}{ }^{2} \mathrm{P}_{3 / 2} \\
\lambda=183.18 \AA \\
2-10\end{array}$} & 5 & $8.539 \mathrm{E}-03$ & \multirow{6}{*}{$\begin{array}{c}2 \mathrm{~s}^{2} 2 \mathrm{p}^{2} \mathrm{P}_{3 / 2}^{\circ}-2 \mathrm{~s} 2 \mathrm{p}^{2}{ }^{2} \mathrm{D}_{5 / 2} \\
\lambda=253.79 \AA \\
2-7\end{array}$} & 5 & $8.857 \mathrm{E}-03$ \\
\hline & 10 & $6.207 \mathrm{E}-03$ & & 10 & $6.911 \mathrm{E}-03$ \\
\hline & 15 & $5.178 \mathrm{E}-03$ & & 15 & $5.934 \mathrm{E}-03$ \\
\hline & 20 & $4.563 \mathrm{E}-03$ & & 20 & $5.324 \mathrm{E}-03$ \\
\hline & 25 & $4.139 \mathrm{E}-03$ & & 25 & $4.893 \mathrm{E}-03$ \\
\hline & 50 & $3.038 \mathrm{E}-03$ & & 50 & $3.728 \mathrm{E}-03$ \\
\hline \multirow{6}{*}{$\begin{array}{c}2 \mathrm{~s}^{2} 2 \mathrm{p}^{2} \mathrm{P}_{3 / 2}^{\circ}-2 \mathrm{~s} 2 \mathrm{p}^{2}{ }^{2} \mathrm{P}_{1 / 2} \\
\lambda=\underset{2-9}{187.16 \AA} \\
2-9\end{array}$} & 5 & $8.963 \mathrm{E}-03$ & \multirow{6}{*}{$\begin{array}{c}2 \mathrm{~s}^{2} 2 \mathrm{p}^{2} \mathrm{P}_{3 / 2}^{\circ}-2 \mathrm{~s} 2 \mathrm{p}^{2}{ }^{2} \mathrm{D}_{3 / 2} \\
\lambda=254.64 \AA \\
2-6\end{array}$} & 5 & $1.827 \mathrm{E}-02$ \\
\hline & 10 & $6.509 \mathrm{E}-03$ & & 10 & $1.454 \mathrm{E}-02$ \\
\hline & 15 & $5.417 \mathrm{E}-03$ & & 15 & $9.105 \mathrm{E}-03$ \\
\hline & 20 & $4.760 \mathrm{E}-03$ & & 20 & $7.081 \mathrm{E}-03$ \\
\hline & 25 & $4.305 \mathrm{E}-03$ & & 25 & $6.073 \mathrm{E}-03$ \\
\hline & 50 & $3.128 \mathrm{E}-03$ & & 50 & $5.445 \mathrm{E}-03$ \\
\hline
\end{tabular}


[3] Werner K, Rauch T, Knörzer M, Kruk J W. First detection of bromine and antimony in hot stars. A\&A 2018;614:A96. doi:10.1051/00046361/201732723-18.

[4] Rauch T, Werner K, Quinet P, Kruk J W. New Ba v, Bavi, and Bavii oscillator strengths and the barium abundance in the hot white dwarfs G191-B2B and RE0503-289. A\&A 2014;566:A10. doi:10.1051/00046361/201423878.

[5] Rauch T, Quinet P, Knörzer M, Hoyer D, Werner K, Kruk J W, Demleitner M. New Se V, Sr IV-VII, Te VI, and I VI oscillator strengths and the Se, $\mathrm{Sr}$, Te, and I abundances in the hot white dwarfs G191-B2B and RE0503289. A\&A 2017;606:A105. doi:10.1051/0004-6361/201730383.

[6] Nikiforov A Yu, Leys Ch., Gonzalez M A, Walsh J. L. Electron density measurement in atmospheric pressure plasma jets: Stark broadening of hydrogenated and non-hydrogenated lines. Plasma Sources Sci. Technol. 2015;24:034001. doi:10.1088/0963-0252/24/3/034001.

[7] Barstow M A, Hubeny I, Holberg J B. The effect of photospheric heavy elements on the hot DA white dwarf temperature scale. MNRAS 1998;299:520-534. doi:10.1046/j.1365-8711.1998.01794.x.

[8] Werner K. On the Balmer Line Problem. Astrophys. J. Lett. 1996;457:L39. doi:10.1086/309889.

[9] Zhang C, Ping L I, Xiao-Hui D. Energy levels of highly ionized Ar XIV. Commun. Theor. Phys. (Beijing, China) 2006;46:723-726. doi:10.1088/0253-6102/46/4/031.

[10] Wang J-S, Griem H R, Rowan W L. Ionization and recombination rates for argon ions Ar XII-XVI from time-resolved spectroscopy of tokamak plasmas. Phys. Rev. A 1987;36:951-954. doi:10.1103/PhysRevA.36.951.

[11] Lepson J K, Beiersdorfer P, Behar E, Kahn S M. Emission-line spectra of Ar IX-Ar XVI in the soft X-ray region 20-50 А. Astrophys. J. 2003;590:604-617. doi:10.1086/374980.

[12] Fawcett B C, Gabriel A H, Paget T M. New observations of the spectra of $\operatorname{argon} \mathrm{X}$ to $\mathrm{XV}$ and of isoelectronic emission lines in silicon VII to $\mathrm{X}$, Phosphorus X, sulphur IX to XII and chlorine X to XIV. J. Phys. B: At. Mol. Phys. 1971;4:986-994. doi:10.1088/0022-3700/4/7/014.

[13] Connerade J P, Peacock N J, Speer R J. The spectrum of multiply ionized argon between 20 and 40 angstroms. Sol. Phys. 1971;18:63-71. doi:10.1007/BF00146033.

[14] Fawcett B C, Gabriel A H, Jones B B, Peacock N J. Grazing incidence spectra of highly ionized atoms from laboratory plasmas. Proc. Phys. Soc. 1964;84:257-338. doi:10.1088/0370-1328/84/2/308.

[15] Fawcett B C, Gabriel A H. Resonance lines of argon IX and X. Proc. Phys. Soc. 1964;84:1038-1040. doi:10.1088/0370-1328/84/6/124.

[16] Deutschman W E, House L L. Additional resonance lines of highly ionized sulfur, chlorine, argon, and potassium. Astrophys. J. 1967;149:451452. doi:10.1086/149273

[17] Buchet J P, Bouchet-Poulizac M C, Denis A, Desesquelles J, Do Cao G. Beam-foil spectroscopy of highly ionized argon. Phys. Scr. 1974;9:221225. doi:10.1088/0031-8949/9/4/007.

[18] Bhatia A K, Seely J F, Feldman U. Atomic data and spectral line intensities for the nitrogen isoelectronic sequence (Ar XII through $\mathrm{Kr}$ XXX). At. Data Nucl. Data Tables 1989;43:99-143. doi:10.1016/0092640X(89)90016-8.

[19] Bhatia A K, Feldman U, Seely J F. Atomic data and spectral line intensities for the boron isoelectronic sequence (Ar XIV through $\mathrm{Kr}$ XXII). At Data Nucl. Data Tables 1986;35:319-343. https://doi.org/10.1016/0092640X(86)90025-2.

[20] Zhang H L, Sampson D H. Relativistic distorted-wave collision strengths and oscillator strengths for the $105 \Delta \mathrm{n}=0$ transitions with $\mathrm{n}=2$ in the $81 \mathrm{~N}$ like ions with $12 \leq Z \leq 92$. At. Data Nucl. Data Tables 1999;72:153-216. doi:10.1006/adnd.1999.0812.

[21] Eissner W, Landi E, Bhatia A K. Atomic data and spectral line intensities for Ar XII. At. Data Nucl. Data Tables 2005;89:139-194. doi:10.1016/j.adt.2005.02.001.

[22] Rynkun P, Jönsson P, Gaigalas G, Froese Fischer C. Energies and E1, M1, E2, M2 transition rates for states of the $2 s^{2} 2 p, 2 s 2 p^{2}$, and $2 p^{3}$ configurations in boron-like ions between N III and Zn XXVI. At. Data Nucl. Data Tables 2012;98:481-556. doi:10.1016/j.adt.2011.08.004.

[23] Wang K, Si R, Dang W, Jönsson P, Guo X L, Li S, Chen Z B, Zhang H, Long F Y, Liu H T, Li D F, Hutton R, Chen C Y, Yan J. Calculations with spectroscopic accuracy: energies and transition rates in the nitrogen isoelectronic sequence from Ar XII to Zn XXIV. Astrophys. J., Suppl. Ser. 2016;223:3(33pp). doi:10.3847/0067-0049/223/1/3.
[24] Mattioli $\mathrm{M}$ et al. Experimental and simulated argon spectra in the 2.3$3.4 \mathrm{~nm}$ region from tokamak plasmas. J. Phys. B: At. Mol. Opt. Phys. 2001;34: 127-142. doi:10.1088/0953-4075/34/2/301.

[25] Elabidi H, Ben Nessib N, Sahal-Bréchot S. Quantum mechanichal calculations of the electron-impact broadening of spectral lines for intermediate coupling. J. Phys. B: At. Mol. Opt. Phys. 2004;37:63-71. doi:10.1088/0953-4075/37/1/004.

[26] Elabidi H, Ben Nessib N, Cornille M, Dubau J, Sahal-Bréchot S. Electron impact broadening of spectral lines in Be-like ions: quantum calculations. J. Phys. B: At. Mol. Opt. Phys. 2008;41:025702. doi:10.1088/09534075/41/2/025702.

[27] Elabidi H, Sahal-Bréchot S, Ben Nessib N. Quantum Stark broadening of $3 \mathrm{~s}-3 \mathrm{p}$ spectral lines in Li-like ions; Z-scaling and comparison with semi-classical perturbation theory. EPJD 2009;54:51-64. doi:10.1140/epjd/e2009-00167-8.

[28] Elabidi H, Ben Nessib N, Sahal-Bréchot S. Electron impact broadening of $\mathrm{Si}$ IV spectral lines: Comparison with recent experiments. J. Quant. Spectrosc. Radiat. Transfer 2012;113:1606-1611. doi:dx.doi.org/10.1016/j.jqsrt.2012.03.028

[29] Bukvić S, Djeniže S, Srećković A. Line broadening in the Si I, Si II, Si III, and Si IV spectra in the helium plasma. A\&A 2009;508:491-500. doi:10.1051/0004-6361/200912046.

[30] Aloui R, Elabidi H, Sahal-Bréchot S, Dimitrijević M S. Quantum and semiclassical Stark widths for Ar VII spectral lines. Atoms 2018;6:20. doi:10.3390/atoms6020020.

[31] Elabidi H, Sahal-Bréchot S. Quantum mechanical Stark widths for Ar V and Ar VI lines: scaling with temperature. Mon. Not. R. Astron. soc., MNRAS 2018;480:697-706. doi:10.1093/mnras/sty1858.

[32] Elabidi H, Sahal-Bréchot S. Stark line widths for N-like ions Na V, Mg VI, Al VII, and Si VIII. Z-scaling. MNRAS 2019;484:1072-1078. doi:10.1093/mnras/sty3516.

[33] Aloui R, Elabidi H, Hamdi R, Sahal-Bréchot S. Quantum Stark broadening data for Ar VIII and Ar IX lines. MNRAS 2019:484;4801-4810. doi:10.1093/mnras/stz303.

[34] Eissner W, Jones M, Nussbaumer H. Techniques for the calculation of atomic structure and radiative data including relativistic corrections. Comput. Phys. Commun. 1974;8:270-306. doi:10.1016/00104655(74)90019-8.

[35] Eissner W. The UCL distorted wave code. Comput. Phys. Commun. 1998;114:295-341. doi:10.1016/S0010-4655(98)00082-4.

[36] Saraph H E. Fine structure cross sections from reactance matrices-a more versatile development of the program jajom. Comput. Phys. Commun. 1978;15:247-258. doi:10.1016/0010-4655(78)90095-4.

[37] Bethe H A, Salpeter E E. Quantum mechanics of one-and two-electron atoms. New York: Academic Press, 1957.

[38] Kramida, A., Ralchenko, Yu., Reader, J., and NIST ASD Team (2018). NIST Atomic Spectra Database (ver. 5.5.6), [Online]. Available: https://physics.nist.gov/asd [2018, July 25]. National Institute of Standards and Technology, Gaithersburg, MD.

[39] Edlén B. Comparison of theoretical and experimental level values of the $n=2$ configurations in the nitrogen isoelectronic sequence. Phys. Scr. 1984;30:135-145. do:10.1088/0031-8949/30/2/007.

[40] Liang G Y, Badnell N R, Zhao G. R-matrix electron-impact excitation data for B-like isoelectronic sequence. A\&A 2012;547:A87. doi:10.1051/0004-6361/201220277.

[41] Badnell N R. A Breit-Pauli distorted wave implementation for AUTOSTRUCTURE. Comput. Phys. Commun. 2011;182:15281535.doi:10.1016/j.cpc.2011.03.023.

[42] Aggarwal K M, Keenan F P, Nakazaki S. Energy levels and radiative rates for transitions in Ar XIII, Ar XIV AND Ar XV. A\&A 2005;436:11411150. doi:10.1051/0004-6361:20052847.

[43] Dyall K G, Grant I P, Johnson C T, Parpia F A, Plummer E P. GRASP: A general-purpose relativistic atomic structure program. Comput. Phys. Commun. 1989;55:424-456. doi:10.1016/0010-4655(89)90136-7.

[44] Merkelis G, Vilkas M J, Kisielius R, Gaigalas G, Martinson I. Electric dipole transitions in ions of the N I isoelectronic sequence. Phys. Scr. 1997;56,41-55. doi:10.1088/0031-8949/56/1/007.

[45] Grant I P. Relativistic Quantum Theory of Atoms and Molecules. Springer, Science-Business Media, New York 2007.

[46] Jönsson P, He C, Froese Fischer C, Grant I P. The grasp2K relativistic atomic structure package. Comput. Phys. Commun. 2007;177:597-622. 
doi:10.1016/j.cpc.2007.06.002. 
$\underline{\text { Conflict of Interest: }}$

Dear Editor;

The authors declare no conflict of interest in this paper.

Thank you

Sincerely,

Rihab Aloui, Haykel Elabidi and Sylvie Sahal-Bréchot 